\title{
A cloud service platform integrating additive and subtractive manufacturing with high resource efficiency
}

Cheng Qian, Yingfeng Zhang, Yang Liu and Zhe Wang

The self-archived postprint version of this journal article is available at Linköping University Institutional Repository (DiVA):

http://urn.kb.se/resolve?urn=urn:nbn:se:liu:diva-161368

N.B.: When citing this work, cite the original publication.

Qian, C., Zhang, Y., Liu, Y., Wang, Z., (2019), A cloud service platform integrating additive and subtractive manufacturing with high resource efficiency, Journal of Cleaner Production, 241, UNSP 118379. https://doi.org/10.1016/j.jclepro.2019.118379

Original publication available at:

https://doi.org/10.1016/j.jclepro.2019.118379

Copyright: Elsevier

http://www.elsevier.com/

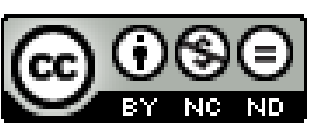




\section{Journal Pre-proof}

A cloud service platform integrating additive and subtractive manufacturing with high resource efficiency

Cheng Qian, Yingfeng Zhang, Yang Liu, Zhe Wang

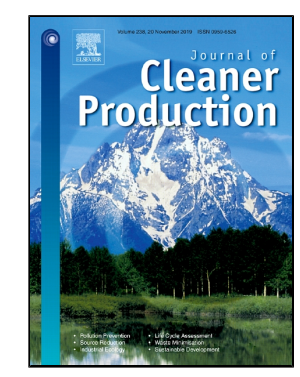

PII:

S0959-6526(19)33249-4

DOI:

https://doi.org/10.1016/j.jclepro.2019.118379

Article Number:

118379

Reference:

JCLP 118379

To appear in:

Journal of Cleaner Production

Received Date:

24 October 2018

Accepted Date:

09 September 2019

Please cite this article as: Cheng Qian, Yingfeng Zhang, Yang Liu, Zhe Wang, A cloud service platform integrating additive and subtractive manufacturing with high resource efficiency, Journal of Cleaner Production (2019), https://doi.org/10.1016/j.jclepro.2019.118379

This is a PDF file of an article that has undergone enhancements after acceptance, such as the addition of a cover page and metadata, and formatting for readability, but it is not yet the definitive version of record. This version will undergo additional copyediting, typesetting and review before it is published in its final form, but we are providing this version to give early visibility of the article. Please note that, during the production process, errors may be discovered which could affect the content, and all legal disclaimers that apply to the journal pertain.

(C) 2019 Published by Elsevier. 


\title{
A cloud service platform integrating additive and subtractive manufacturing with high resource
}

\section{efficiency}

\author{
Cheng Qian ${ }^{\mathrm{a}, \mathrm{c}}$, Yingfeng Zhang a *, Yang Liu ${ }^{\mathrm{b}, \mathrm{d}}{ }^{*}$, Zhe Wang a \\ a Key Laboratory of Contemporary Design and Integrated Manufacturing Technology, Ministry of Education, Northwestern \\ Polytechnical University, Shaanxi, P. R. China, 710072 \\ ${ }^{b}$ Department of Management and Engineering, Linköping University, SE-581 83 Linköping, Sweden \\ ${ }^{c}$ Department of Mechanical and Energy Engineering, Southern University of Science and Technology, Shenzhen, \\ Guangdong 518055, P. R. China \\ ${ }^{\mathrm{d}}$ Department of Production, University of Vaasa, 65200 Vaasa, Finland \\ * Corresponding Author: zhangyf@nwpu.edu.cn (Y. Zhang), yang.liu@liu.se (Y. Liu)
}

\begin{abstract}
Cloud manufacturing has been studied for years, yet commercial implementations are still limited. The recent advances in information technology have stimulated the free sharing of additive and subtractive manufacturing (A/SM) resources through cloud services. Currently, due to the lack of a general method to model manufacturing capabilities, as well as the absence of an open platform to integrate business and manufacturing processes, it is difficult to integrate $\mathrm{A} / \mathrm{SM}$ resources within one platform efficiently and seamlessly. In this research, a service encapsulation model for A/SM resources was described using ontology modeling technique. A collaborative cloud platform integrating A/SM was designed that can provide optimal production plans considering time, cost, quality, and energy waste during manufacturing. The proposed platform and models were demonstrated by a prototype system and tested in a case study, which shows the integrated platform can increase the utilization rate of resources while reducing energy consumption. This research has provided a practical tool for virtualization, integration, and configuration of A/SM resource with high efficiency.
\end{abstract}

Keywords: Cloud manufacturing, additive manufacturing, subtractive manufacturing, resource efficiency

\section{Introduction}

Additive manufacturing (AM, e.g. 3D printing) has attracted enormous attention from multiple fields, including the manufacturing industry (Chua and Leong, 2014). Featured with its single-step fabrication method (Lee et al., 2016), additive manufacturing can produce models with complicated interior structures, greatly simplifying the complexity of manufacturing process route planning and shop floor scheduling. This simplification has brought a brand-new way of rapid prototyping for designers. Applications of additive manufacturing can be found in industries like automobile (Rahim and Maidin, 2014), biomedical (Stansbury and Idacavage, 2016), construction (Li et al., 2017), etc. Both academia and industry are trying to increase the reliability, repeatability, and efficiency of additive manufacturing so that they can be well applied to fabricate not only prototypes but also final products (Lee et al., 2016). Recently, a hybrid process which integrates both additive and subtractive manufacturing (A/SM) has been studied to take advantage of the different manufacturing methods. The literature on resource-efficiency optimization and energy-consumption modeling can be found in fields of both additive and subtractive manufacturing (Peng, 2016; Simeone et al., 2018). However, most research only focused on modeling the individual additive manufacturing resources and they have not studied the resource configuration of a platform that integrates $\mathrm{A} / \mathrm{SM}$ resources. When solving the resource configuration problem of a platform that includes multiple A/SM resources, despite the locations of distributed resources, some other factors should also be considered to realize efficient and clean production. E.g., the heating process of some AM resources can be rather time- and energyconsuming. This process of different AM tasks can be possibly combined or shortened if the required printing temperature of different tasks is considered during resource configuration.

Throughout the development of advanced manufacturing systems such as computer-integrated manufacturing, green manufacturing (Govindan et al., 2015), and industrial product-service systems (IPSS) (Meier et al., 2010), the manufacturing industry has adapted to the trends of servitization (Lee et al., 2014). Cloud manufacturing (CMfg) is an 
Houshmand, 2013). Consequently, CMfg has been extensively researched recently (Tao et al., 2014) by using serviceoriented architecture (SOA) (Valilai and Houshmand, 2013) and some other enabling technologies, such as Internet of things (IoT) and CC (Chard et al., 2012). There are three major parties in CMfg, i.e., (1) the service provider, which could be manufacturing workers, virtualized manufacturing capabilities, or outsourcing resources from third-party companies; (2) the customer, who places the order and initiates the manufacturing process; (3) the cloud platform, which connects the other two roles, organizes production and business process, monitors manufacturing process, and provides necessary statistics or predicted data to its operators.

The previous research on CMfg has been done in different aspects or fields, and some AM platforms are already available online, but some real-world problems still make it difficult for the platforms to support the effective business implementation. For example, it is unclear which parameters should be used to model the 3D printers for CMfg to achieve greater system efficiency and sustainability. Since some production tasks require both additive and subtractive manufacturing processes, their parameters used for modeling the resource capability on the CMfg platform should be compatible with each other. Thus, a general structure for modeling the capability of different machines is necessary. Also, the geographically distributed manufacturing resources of the CMfg platform may cause new challenges since the time, cost and energy consumption of logistics within the platform cannot be omitted in the real-world productions. Besides, the coordination of multiple AM resources needs to be considered for better resource efficiency. This research addresses some of the problems by introducing an implementation framework for the cloud-based platform of A/SM. The primary research questions are listed as follows.

(1) It is unclear how to encapsulate the manufacturing machines in a general method so that they can provide services and be identified through a cloud platform. The ability of the A/SM resources to collect certain data from the manufacturing environment should be firstly guaranteed, which is a basic requirement for any machines that can be monitored and adjusted in real time through a cloud platform. Also, the type of parameters used in modeling the manufacturing capabilities is vital to the reliability, efficiency, and compatibility of the CMfg system.

(2) The establishment of the collaborative cloud platform should be carefully studied according to the business and manufacturing processes of A/SM. Since AM does not involve many complex manufacturing routes compared to the traditional ones, but it does include some new processes like the verification and correction of the models in the submitted tasks, or the joint collaboration with some computer-numerical-control (CNC) machines, the cloud platform should take advantage of its service-oriented feature to support the new manufacturing processes. It is also challenging for the CMfg platform to integrate the $\mathrm{A} / \mathrm{SM}$ processes in a reasonable way.

(3) It should be further analyzed that how to improve the service models to address the real-world problems that prevent the effective implementation of CMfg. Some hidden cost of time and energy should be addressed, e.g. if the sequence of processes is not carefully designed, replacing the clamps between processes may be necessary, which will increase the idling time of machine; similarly, adjusting the temperature of 3D printers' heating bed and extruder for different tasks may cost extra time and energy. Besides, the distance-related constraints should be considered as the chosen set of resources may be far away from each other and the logistics may take up much time.

Based on these research questions, this research designed a general platform for the distributed A/SM resources considering the process constraints of the tasks and the location constraints of the resources. The AM resources were virtualized and encapsulated as cloud manufacturing services first before being published to the CMfg platform. By integrating the techniques of predictive scheduling, production-logistics synchronization, etc., CMfg is more likely to be commercially implemented in more areas and fields. Such implementations are directly or indirectly beneficial to the development of economy, society, and environmental protection. The distributed resources can be invoked online by a wider range of customers, increasing the utilization rate of the individual A/SM resources while reducing the unnecessary consumption of the resources that are rarely used. Besides, the platform will provide some simple tools for the A/SM resource encapsulation, giving more market opportunities to the small business operators, and stimulating the fair competition of industry. The proposed models in this research help to further promote clean production in cloud manufacturing. 
The remainder of this paper is organized as follows. Related works on $\mathrm{CMfg}$ and service encapsulation were reviewed

in Section 2. Section 3 presented the overall architecture for A/SM based on cloud services, which includes the introduction to the collaborative cloud platform, plus with the business and manufacturing processes of A/SM. Section 4 described an encapsulation and virtualization model for the Fused Deposition Modeling (FDM) 3D printers, which can also be adapted to model the subtractive manufacturing machines. Section 5 discussed some improved strategies of the CMfg platform to increase resource efficiency while balancing the time, cost, and quality of the production. These strategies and models were tested and analyzed in some case scenarios in Section 6. Section 7 summarized the paper and proposed some future research directions.

\section{Literature review}

A literature survey has been conducted to reveal the development of CMfg, including its key enabling technologies, the features of the currently implemented platforms, and the major drawbacks when establishing a CMfg platform for A/SM.

\subsection{The development of cloud manufacturing and some enabling technologies}

CMfg emerges from the advancement in the manufacturing management methods (e.g. the just-in-time mode), the development of manufacturing paradigms (e.g. computer-integrated manufacturing, agile manufacturing, etc.), and the implementation of enterprise information technologies (e.g., CC, IoT, SOA, etc.) (Zhang L. et al., 2014). In CMfg, traditional manufacturing resources and capabilities are virtualized as manufacturing services, so as to simplify the invoking and sharing of those resources and capabilities (Xu, 2012). The service provider, the service platform, and the customer are the primary parties in CMfg, and the interrelations among them were analyzed by Ren et al. (2013). Generally speaking, there are four categories of CMfg models, namely the private, community, public, and hybrid cloud models (Buckholtz et al., 2015). Research directions of CMfg include the systematic architecture design of CMfg (Tao et al., 2014), the design of services (Zheng et al., 2018), the management of services in CMfg (Tao et al., 2015), the resourceefficiency optimization in CMfg (Simeone et al., 2019), the implementation of the cloud platform to match the tasks with services (Argoneto and Renna, 2016), etc. The energy management of the platforms was also discussed by Ma et al. (2019).

Manufacturing resources and capabilities need to be encapsulated in a unified way to become manufacturing services. A lot of information technologies have been applied to fulfill this requirement, such as the semantic web (Dadzie et al., 2009), ontology modeling (Bhattacharya et al., 2012), the Universal Description, Discovery and Integration (UDDI) technique (Curbera et al., 2002), etc. To be specific, an IoT-based framework for real-time information capturing and integration was proposed by Zhang Y. et al. (2014), so that the real-time status of manufacturing resources can be reached by the cloud platform and customers. The Web Ontology Language (OWL) was applied to extract the manufacturing characteristics (Kang et al., 2015), and the manufacturing capabilities were encapsulated by using the Manufacturing Service Description Language (MSDL) (Ameri and McArthur, 2014). Then, the model of manufacturing services could be established with UDDI (Jang et al., 2008). To improve the efficiency of service discovery, a hyperlink based global social network was formed by Chen and Paik (2013). The encapsulated additive or subtractive manufacturing resources are turned into online services and can be invoked through the CMfg platform. The matching, evaluation, selection, and configuration of services were summarized by Liu et al. (2018).

2.2 The current cloud platforms and the integration of additive and subtractive manufacturing

Research on the implementation of CMfg can be seen in different phases of manufacturing, including cloud-based design, cloud-based manufacturing, cloud remanufacturing, etc. (Wu et al., 2013; Caggiano, 2018; Wang et al., 2014). Wu et al. (2013) summarized the four main types of services in CMfg i.e. the Hardware-as-a-service (Haas), Software-as-aservice (Saas), Platform-as-a-service (Paas), and Infrastructure-as-a-service (Iaas). They also studied a number of implementation architectures, business models, existing cases of CMfg, etc., where most existing commercial cases focused on the customization in product design and assembly, and sharing the spared manufacturing resources online.

Compared to the subtractive manufacturing resources (e.g. CNC machines), the recently emerged AM resources are generally born with sufficient capabilities to capture the environment information and to be controlled digitally due to the 
built-in sensors and actuators. Consequently, the cloud platforms designed for AM resources can be easily found in many theoretical studies and business cases. Managing distributed 3D printing resources in CMfg for customized products was studied by Mai et al. (2016), where they listed different types of services and analyzed the basic processes of 3D printing services in CMfg. Brant et al. (2015) studied the production performance of a cloud-based additive manufacturing system for metal structures in terms of the execution accuracy of the instructions, the system reliability of the on-demand control, the data availability of the on-demand feedback, the production time and efficiency, etc. Rudolph and Emelmann (2017) proposed a pricing and order processing mechanism for additive manufacturing on cloud platforms, and compared the different production cost for parts when using different manufacturing technologies (i.e. 3D printing, CNC milling, and casting). Paris et al. (2016) made a similar comparison from the environmental impact perspective.

Since both additive and subtractive manufacturing resources can be encapsulated and published to the CMfg platform, some researchers recently focused on the relations between cloud-based A/SM resources and the possibility of merging the two different kinds of manufacturing method together. Watson and Taminger (2018) proposed a decision support model for the selection between A/SM resources on a platform according to the energy consumption of the different methods. Priarone and Ingarao (2017) modeled the sustainability of the pure subtractive versus the A/SM approaches and provided the criteria for an eco-friendlier decision-making process. Du et al. (2016) conducted an experimental study that combined the $\mathrm{A} / \mathrm{SM}$ resources, which simplified the overall process route while guaranteed the precision of certain structures. Newman et al. (2015) also proposed a process planning method where A/SM approaches were used together, and this idea was extended to the remanufacturing field by Le et al. (2017).

\subsection{Limitations of the current models and platforms}

There are some major drawbacks in the existing CMfg platforms that prevent them from expanding in the market. Firstly, the current research on CMfg platforms that integrates A/SM resources is limited. A lot of cloud-based 3D printing service providers can be found online while little supported the collaboration with milling, turning, and other subtractive processing approaches. Thus, an in-depth study on the modeling of A/SM resources should be carried out so that the CMfg platform is compatible with most resources. Secondly, a practical, economical, and environmental efficient tool that turns the customers' orders into manufacturing tasks or instructions for the distributed A/SM resources is missing. The current decision-support models for choosing additive or $\mathrm{A} / \mathrm{SM}$ resources generally focuses on the economic and environmental performance of the different manufacturing approaches, they were not being widely applied partly because some were inaccurate in estimating the economic and environmental cost (e.g., the internal logistics between the distributed A/SM resources were sometimes ignored, which may cost extra time and energy), partly because some decision-support criteria were too ideal for customers and platform operators (e.g. customers will probably not chose the additive manufacturing approach for building a part that is in urgent need compared to milling, where the former one is more environmentally friendly but takes longer time). Consequently, a feasible mechanism for process scheduling and resource matching is required to bridge the gap between theoretical methods and business practice.

\section{The overall architecture for the cloud manufacturing service-based additive and subtractive}

\section{manufacturing}

AM has changed the traditional manufacturing process greatly. Thus, the CMfg platform for A/SM needs to be redesigned according to the business and manufacturing processes. Thanks to the service-oriented architecture, the primary modification required for cloud $\mathrm{A} / \mathrm{SM}$ is to change the services, as compared to a traditional CMfg platform. In this section, a collaborative platform for cloud $\mathrm{A} / \mathrm{SM}$ is designed, and the new manufacturing process is analyzed.

3.1 Collaborative platform for the cloud-based additive and subtractive manufacturing resources 


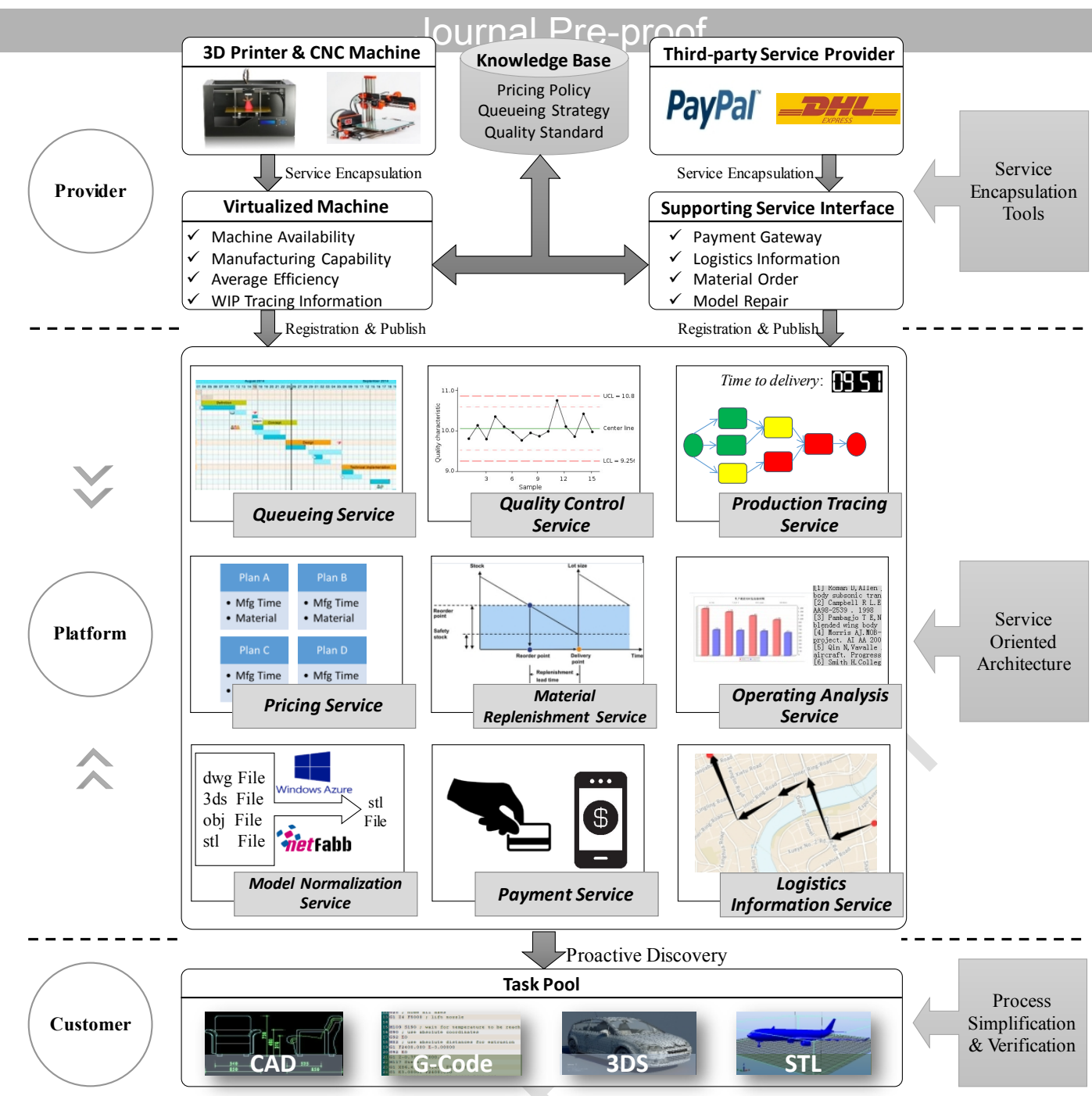

Fig. 1 Collaborative platform for the cloud-based additive and subtractive manufacturing resources (CNC: computer-numerical-control; WIP: work-in-process).

The designed platform is shown in Fig. 1. The upper part represents the service provider, the middle part illustrates the CMfg platform, and the customer is demonstrated at the bottom. AM resources (e.g. 3D printers) and subtractive manufacturing resources (e.g. CNC machines) are encapsulated as manufacturing services before being published to CMfg platform. Additionally, third-party service providers are involved to help with the business processes of cloud AM. Service interfaces provided by them are also registered in the CMfg platform. In this research, manufacturing capabilities provided by the 3D printers and $\mathrm{CNC}$ machines are defined as $\mathrm{A} / \mathrm{SM}$ services, which directly focus on the manufacturing execution processes (i.e. part fabrication) and are described in Section 4. Other services that are involved in the manufacturing or business processes are called the auxiliary manufacturing services on this platform, which extend the capability of the cloud platform so that the $\mathrm{A} / \mathrm{SM}$ resources can be monitored, controlled, and configured timely and can process the orders autonomously. These services are further discussed in Section 5. The knowledge base aims at value-adding to the raw data from the manufacturing environment. They define the manufacturing rules and the standard business procedures such as the quality standard, the pricing policy, the queueing strategy, etc. These data may have different accessibility according to the specific system design to guarantee the stability, security, efficiency, and intelligent of the platform. E.g., the platform may invoke some models in a Saas manner, such as the one proposed by Zhu et al. (2018), which can be used to optimize the control parameters of 3D printers and improve the manufacturing quality through machine learning. The two types of services are stored on the CMfg platform, taking and analyzing orders, creating processing instructions for the $\mathrm{A} / \mathrm{SM}$ resources, monitoring manufacturing execution processes, coordinating distributed resources, processing transactions and providing tracing logs of production, etc. Customers can submit tasks to the task pool. When submitting the task, they need to clarify the unique requirement for their task, e.g., what is the latest delivery time, which material should be applied, are there any strength requirements for certain structure, etc. Manufacturing services provided by different A/SM resources will then check their capability properties. Qualified service providers that meet the customers' 
demand will be associated with the task and prepare for subsequent manufacturing and business processes. The complete process is discussed in Section 3.2.

\subsection{Business and manufacturing processes}

The whole process is initiated when a customer submits their production task. They upload their designed drawings to the CMfg platform through encrypted network protocols, plus with manufacturing requirements for their products. The design can be a single component or it may include multiple parts, where the former one will be assigned to one manufacturing resource and the latter will be assigned to one or more resources. In other words, the CMfg platform will not split a single component nor process it on multiple manufacturing resources, which may result in extra processes like assembling the split parts, leading to the change in design and potential defects. The uploaded designs will only be used for the production and will be deleted when the order is completed. However, customers can choose to share their designs on the platform for study or reuse under proper licenses. Since the data transmission and storage is encrypted, and procedures like model analyses and A/SM resource scheduling are completed automatically without human intervention, the possibility of leaking the designs is minimized. (Other security methods may also be applied such as dividing the uploaded files into several blocks that are stored separately.) Upon receiving the task, the model normalization service takes over to convert the file of different formats to the standard model file (e.g., the STL format file). This service will also detect errors and attempt to correct them using CC. The corrections will only happen when the STL format file cannot be analyzed properly or some defects in the polygon mesh for the designs are detected, and will not change the original design. Such defects can be easily detected by the major AM software such as Repetier-host (Repetier, 2016). There are also some commercial and open-sourced tools dedicated to the correction (e.g. Netfabb, Trinckle, MeshFix, etc.). The customers will be notified if such corrections happen and they can reexamine if the updated design files are acceptable.

Following the Paas philosophy, customers only directly interact with the platform rather than the specific processing machines, although they may have access to the production monitoring service. The tasks are manufacturing-approachindependent (i.e. AM, subtractive manufacturing, or a combination of both) as long as they meet all the requirement of geometry, strength, precision, roughness, delivery time, budget, etc. In this regard, customers can provide alternatives that use different manufacturing approaches for the same process. The status of A/SM resources collected by the operating analysis service and the task requirement will be fed to the queuing service, helping to preliminary select the manufacturing approach and decide which resources will potentially take the tasks. Then, the preprocessed model will be sliced for the convenience of AM if necessary. When the preprocessing is done, multiple production plans will be sent to the customers according to the models in Section 5. These plans have different targets and subject to different constraints (e.g., minimized cost, minimized production time, best manufacturing quality, or something in between where some manufacturing parameters can be customized). The customers can, therefore, select a plan and pay for it according to the estimation form the pricing service. Once the payment has been confirmed by the platform, the manufacturing process instructions generated by the queueing service will be sent to $\mathrm{A} / \mathrm{SM}$ resources. The quality control service is responsible to monitor the operations and to reconfigure the resources if necessary. The manufacturing process is accessible to their customers through online graphs, reports, or webcams provided by the production tracing service. If the assembly is required, the operator will assemble the products according to the instructions from the design when all the parts have been printed. Robotic arms or specially certificated operators are also possible options when a sensitive product or a confidential design is involved. Inspections are followed to conduct the overall quality check before delivering the finished products. Customers are accessible to real-time logistics information through the platform and will evaluate the service when they receive the products. Remarks and scores from customers will be stored in the knowledge base and will be analyzed by the operation analysis service. The abovementioned process is summarized in Fig. 2. 


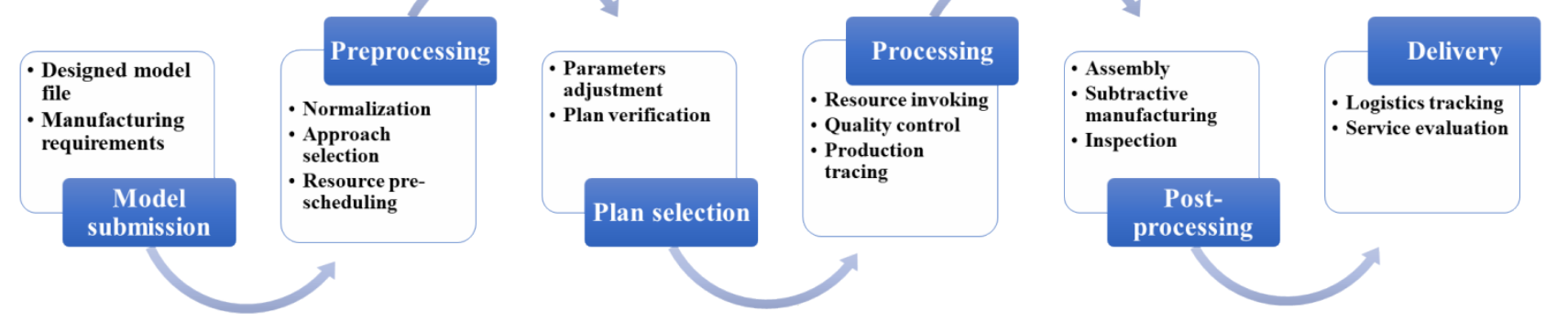

Fig. 2 Business and manufacturing processes of the cloud manufacturing platform.

\section{Encapsulation of manufacturing resources}

To effectively monitor the operations of a manufacturing machine, the encapsulation and virtualization model is applied so that the manufacturing processes become transparent and the servitized resources are accessible at any time. Encapsulation indicates the process to wrap and abstract the physical manufacturing resources into virtualized ones and services, so that they can be invoked and accessed through the platform. Since the ways to model subtractive manufacturing resources such as a CNC machine has been illustrated in the previous work (Zhang et al., 2016), this research will focus on the modeling of AM resources such as a 3D printer. The service encapsulation model discussed in this section enables 3D printers to be virtualized as services before being published to the CMfg platform, so that they can be reached by potential users through the platform.

\subsection{Procedures of encapsulation for 3D printers}

As shown in Fig. 3, three steps are designed to encapsulate the manufacturing capability of 3D printers into cloud services, namely the deployment of 3D printers, the servitization of 3D printers, and the service publishing method.

The first step is to upgrade the traditional facilities to smart manufacturing objects with the capabilities of selfperception and context-awareness. All kinds of information (e.g. the position of the extruder, the temperature of the heating bed, the vibration of the printing surface) are captured by different sensors through the wireless sensor network and are collected for later use, e.g., the motion and temperature control introduced in Section 4.2. The enhancement in the sensing capability of 3D printers helps to provide instant feedback for the manufacturing monitoring and control. The second step is to establish standard models where manufacturing or business activities are encapsulated into web services, and hence the CMfg service-based 3D printers can be realized in a plug-and-play manner by invoking the services. Finally, the service publishing method for 3D printers is applied. By adopting this method, cloud 3D printing services from distributed service providers are registered, published, and accessed through the CMfg platform. The details of these steps are discussed in Section 4.2-Section 4.4.
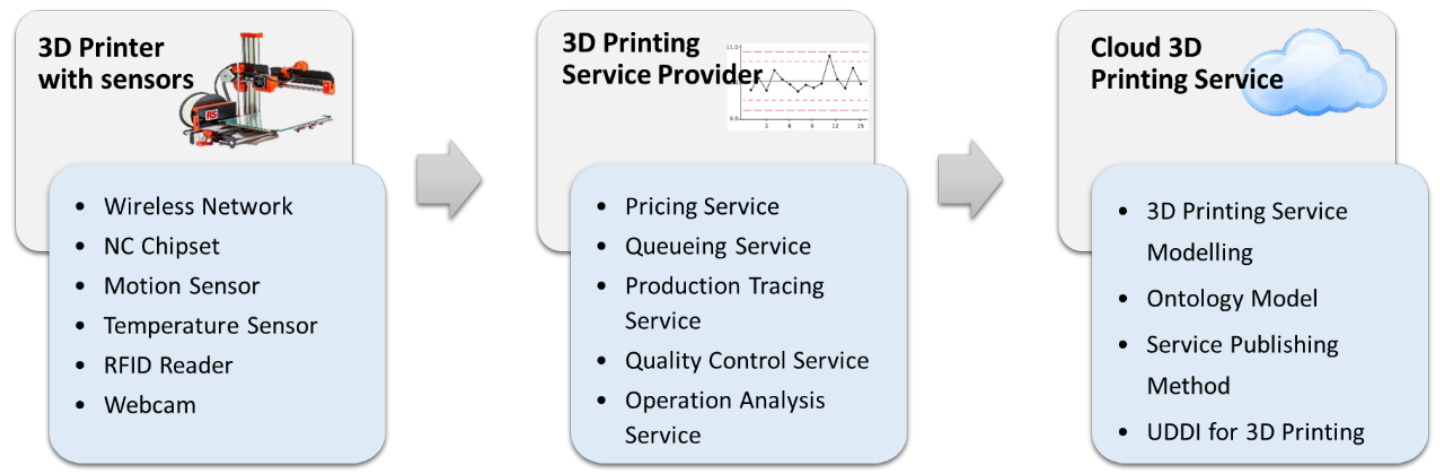

\section{Deployment of} 3D Printers

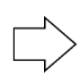

$$
\begin{aligned}
& \text { Servitization of } \\
& \text { 3D Printers }
\end{aligned}
$$
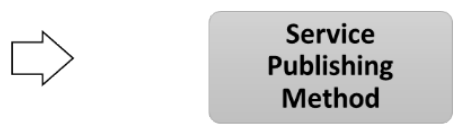

Fig. 3 Procedures of service encapsulation for 3D printers.

(NC: numerical controlled; RFID: radio frequency identification; UDDI: universal description, discovery, and integration)

4.2 Deployment of the cloud 3D printers 
Since 3D printers can be classified into many categories like material extrusion, material jetting, powder bed fusion, etc. (Lee et al., 2016), proper types of the sensor should be chosen and set up when deploying the printers. For example, when dealing with those that print by extruding filament (e.g. the FDM 3D printers), motion sensors and acceleration sensors (which are already embedded in the 3D printers) will detect the position and velocity of the nozzle to fulfill numerical control (NC) of 3D printers. Also, temperature sensors will monitor the temperature of the nozzle, building bed, and core chipsets of the printer for real-time manufacturing control. Radio frequency identification (RFID) readers are arranged to trace the work-in-process (WIP) information of the products. Besides, the webcam is prepared for customers to have a look at the printing progress of their tasks visually. Vibration or even ultrasonic sensors may also be applied for quality control of the products.

After determining the types of sensors, it is necessary to decide how many sensors should be applied, and which are the optimal positions for them. Basically, this is a linear programming problem where sensors should provide all necessary information for manufacturing services like operation analysis and the reliability of sensors is high enough while the cost of them is minimized. By implementing IoT devices, 3D printers can now capture data from their surrounding manufacturing environment timely and precisely.

\subsection{Servitization of 3D printers}

After the configuration of sensors and the network, 3D printers become accessible cloud service providers. Servitization of 3D printers is discussed by analyzing the dual-way data flow from the captured data to manufacturing services, which can be concluded from Fig. 4.

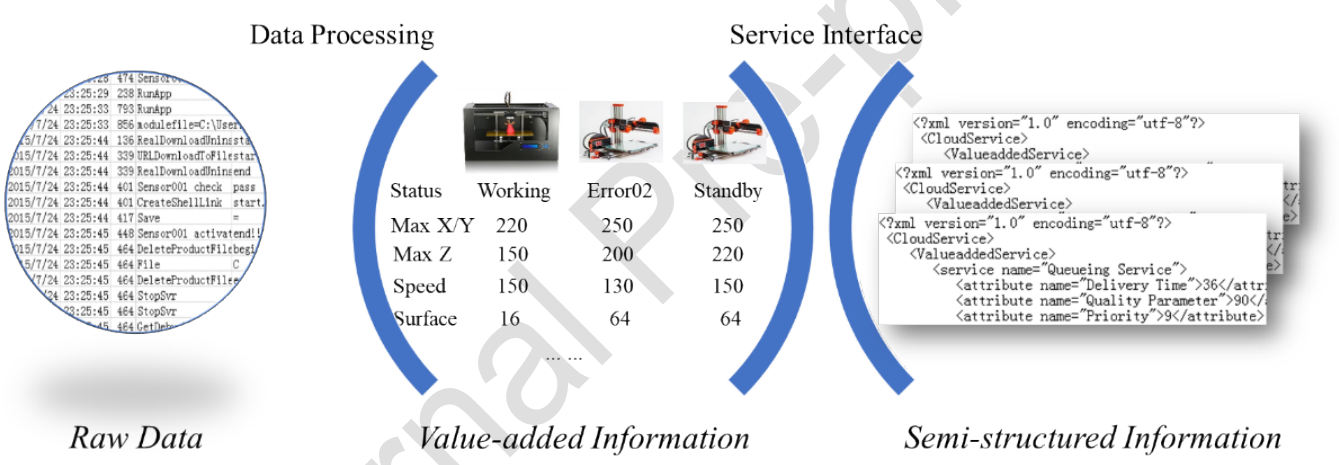

Fig. 4 The different forms of data through manufacturing service modeling.

The real-time manufacturing data includes operating instructions from manufacturing services and data collected from 3D printers. These data streams directly control the production process and reflect the actual manufacturing scenario, but they may be unreadable for customers and operators. Thus, the data processing module is applied to interpret these data as value-added manufacturing information. Based on some mapping mechanism such as manufacturing rules or the characteristics of 3D printers, raw data are wrapped up as properties of an entity or activity. For example, the instant measuring value of one motion sensor reflects the absolute vertical position of the nozzle, and an increased value indicates that the nozzle is moving upwards. The value-added information will then act as input or output values of manufacturing services. After that, manufacturing information are classified into different blocks according to their scopes. Combined with method invocation defined with computer programming languages, those information blocks become service functions with standard inputs and outputs. The service functions are the result of manufacturing service encapsulation and serve as service interfaces of the CMfg platform.

\subsection{Publishing method for manufacturing services}

Manufacturing services can only be accessed by customers and operators after they are registered and published to the CMfg platform. The designed service publishing method provides a universal way to publish services from different service providers. Services can then be accessed through the CMfg platform. Here, UDDI is implemented to realize service publication and discovery. UDDI requires service description and XML-based messaging. A conceptual model of the AM service is established first before being transformed into the ontology model using semantic web technologies. 
Fig. 5 illustrates the conceptual model of the cloud AM service. Five categories of parameters are involved in the model, namely basic properties, capability properties, operating parameters, quality standards, and manufacturing status monitoring information. Basic properties contain information that is used to identify the 3D printer. Inherent characteristics of the printer such as machine ID, age, corresponding operator, and communication configurations are included. Capability properties describe the manufacturing ability and limits of the 3D printers, so that they can take in proper tasks from the CMfg platform respectively. Such read-only properties may include printing size, material restrictions, and printing speed requirement. Operating parameters are responsible for controlling the 3D printers, e.g., what should the extruder and bed temperature be like, how fast the printing will be, is supporting structure necessary, etc. Quality standards also affect the operations of AM, but they especially aim at high Quality of Service (QoS). Time consumption, surface smoothness, structure strength, and customers' subjective satisfaction should all be taken into consideration. Manufacturing status monitoring information is used to track the production progress and to conduct operating analysis. Printing progress, printer's workload, and WIP tracing data become accessible based on this information.

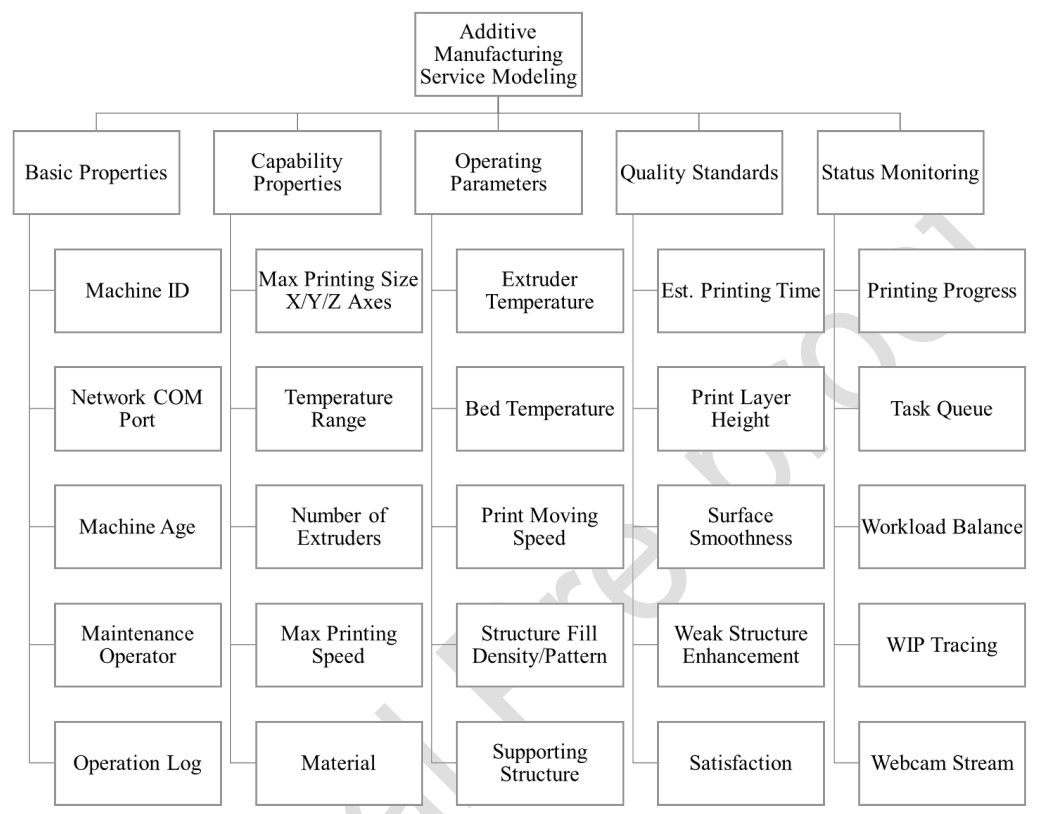

Fig. 5 An example of the conceptual model of cloud-based additive manufacturing services.

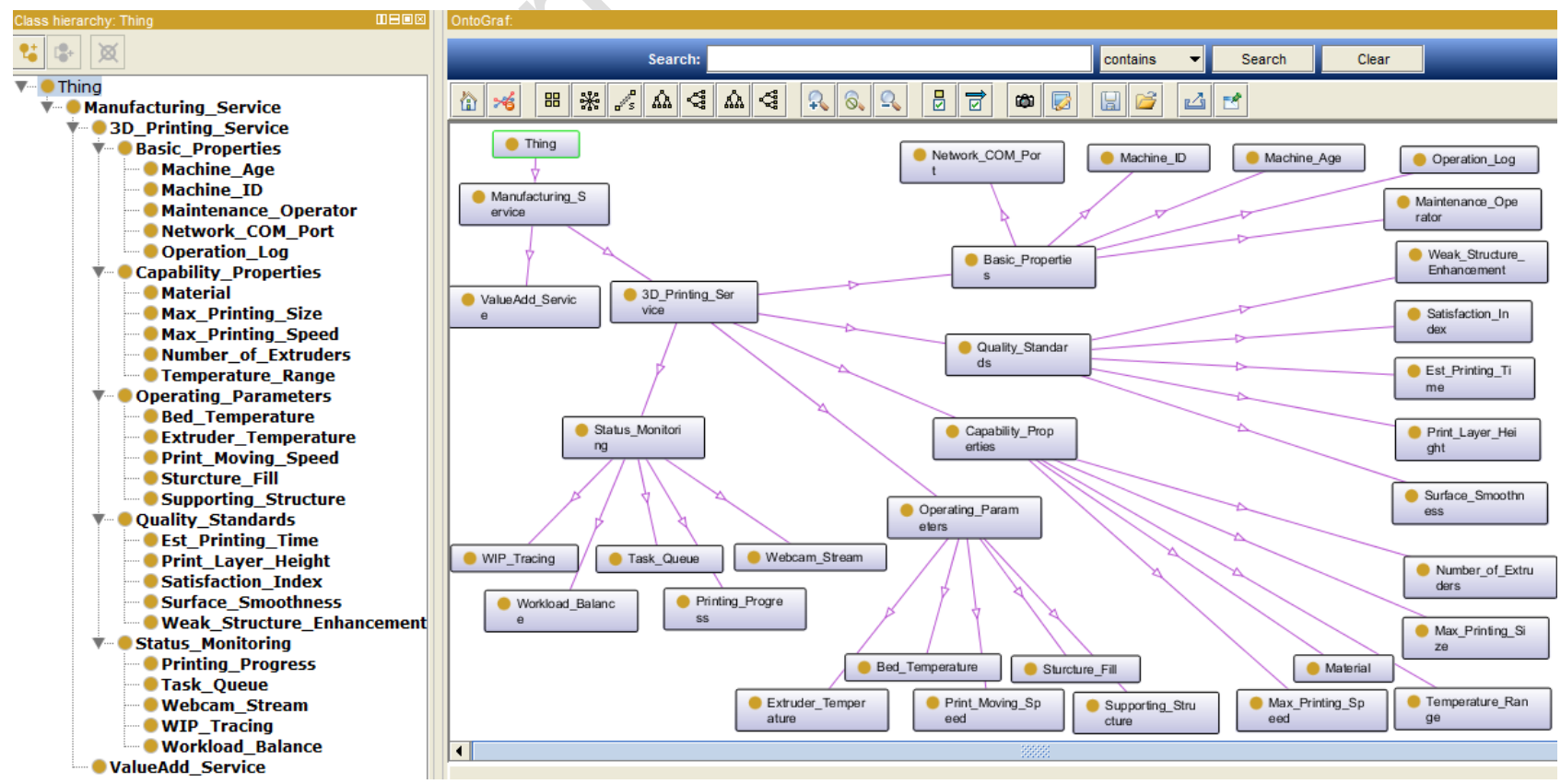

Fig. 6 The ontology model of the additive manufacturing service.

The conceptual model then needs to be translated into ontology models so that they can be understood by the CMfg platform. In this research, Protégé (Stanford Center for Biomedical Informatics Research, 2016) was applied to construct the ontology model, which is shown in Fig. 6. Finally, the ontology model can be registered on the platform using UDDI 

the previous work (Zhang et al., 2016). The procedures introduced in this section can be used to model A/SM resources. Following these steps, the distributed manufacturing resources of different types can be encapsulated and published to the same CMfg platform, which greatly promotes the collaboration between A/SM resources.

\section{An improved strategy for the resource configuration on the platform}

Since the A/SM resources and tasks are registered and published on the cloud platform, it is the platform's responsibility to configure resources in an effective way to satisfy multiple needs from customers. The delivery time, cost, quality of products, etc. are the common criteria for customers to make decisions on a purchase. Fig. 7 illustrates some typical factors that influence the above-mentioned criteria. E.g., The delivery time is jointly decided by the time for manufacturing and transportation. Different processes may be treated at different A/SM resources that can be distant apart from each other, so the transportation time should not be ignored. The adjustable production speed and the set-up time will further decide the manufacturing time.

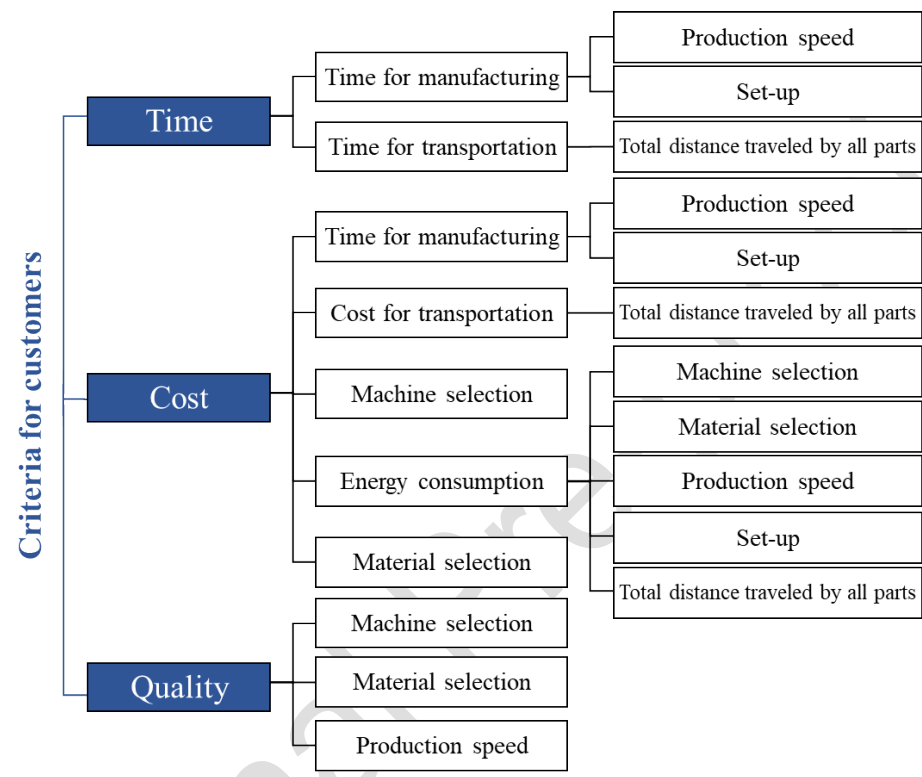

Fig. 7 Factors that influence customers' criteria for selecting the production plan.

The cost of a product is calculated by adding the machine occupation charge (reflecting the duration of the processes that occupy the resources), transportation fees, the basic charge of machines (which is resource-dependent) and material, and the cost of energy consumption. The price leverage is used to encourage customers to select more energy-efficient production plans by carefully modeling the energy consumption and associating it to the pricing service. The detailed models are introduced in Section 5.1.

The quality of a product is generally related to the manufacturing machines, material, and production speed. In FDM additive manufacturing, the production speed is related to the thickness of the printing layers, the speed/acceleration of extruders, the filling structure of printing parts, etc., which may influence the surface roughness, geometric accuracy, structural strength, respectively.

5.1 Formulating the production time and cost

The production time and cost are mathematically modeled in this section. A list of notations is shown in Table 1.

Table 1 Notations of the time, cost, quality models.

\begin{tabular}{cc}
\hline Notation & Description \\
\hline$P_{i}^{m}$ & The $i^{\text {th }}$ process of the $m^{\text {th }}$ task.
\end{tabular}


$T\left(p_{i}, p_{j}\right) \quad$ The logistics time between the $i^{\text {th }}$ and $j^{\text {th }}$ process.

$T_{i}^{m} \quad$ The processing time of $P_{i}^{m}$. Since the production speed is adjustable, $T_{i}^{m} \in\left[T_{i}^{m}(\mathrm{~L}), T_{i}^{m}(\mathrm{U})\right]$.

$t s_{i}^{m} \quad$ The set-up time of $P_{i}^{m}$.

$t c_{i}^{m} \quad$ The converted set-up time of $P_{i}^{m}$.

$c_{i}^{m} \quad$ The cost coefficient of $P_{i}^{m}$ for the machine occupation (per unit time).

$\mathrm{cm}_{i}^{m} \quad$ The material cost of $P_{i}^{m}$.

$C\left(p_{i}, p_{j}\right) \quad$ The cost of transportation between the $i^{\text {th }}$ and $j^{\text {th }}$ process.

$E_{i}^{m} \quad$ The energy consumption for the processing of $P_{i}^{m}$.

$e s_{i}^{m} \quad$ The energy consumption during the set-up for $P_{i}^{m}$.

$e c_{i}^{m} \quad$ The converted energy consumption during the set-up for $P_{i}^{m}$

$E\left(p_{i}, p_{j}\right) \quad$ The energy consumption for transportation between the $i^{\text {th }}$ and $j^{\text {th }}$ process.

$e c_{i}^{m} \quad$ The cost coefficient of the energy consumption on transportation $E\left(p_{i}, p_{j}\right)$.

\subsubsection{Production time}

The production time can be obtained by adding the manufacturing time of all processes (including the set-up time and processing time) and the transportation/logistics time between processes. In this research, delivering a product to the customer is also regarded as a process within the service lifetime, so the location of the customers and the subsequent transportation time can be considered consistently. The production time of task $m$ with $k$ processes (denoted as $T^{m}$ ) is formulated in (1), where $\Sigma T_{i}^{m}, \Sigma t s_{i}^{m}$, and $\Sigma T\left(P_{i}^{m}, P_{i+1}{ }^{m}\right)$ are the processing time, set-up time, and transportation time, respectively.

$$
T^{m}=\sum_{i=1}^{k} T_{i}^{m}+\sum_{i=1}^{k} t s_{i}^{m}+\sum_{i=1}^{k-1} T\left(P_{i}^{m}, P_{i+1}^{m}\right)
$$

Since additive manufacturing is included in this cloud platform, it is pointed out that the heating process (for the extruders and the printing bed) is generally a time-consuming (and energy-consuming) but necessary process during the set-up. The platform is designed to reduce the time and energy consumption during set-up as much as possible by arranging processes that require similar printing temperature close to each other. For example, when a 3D-printing process $\left(P^{\mathrm{A}}\right)$ is arranged right after another printing process $\left(P^{\mathrm{B}}\right)$ on a printer, much time and energy can be saved before the extruders and the printing bed cool down. In another case, if process $P^{\mathrm{A}}$ is inserted into an available time slot of the printer right before process $P^{\mathrm{B}}$, the rewards for saving time (and energy) is also reflected on $P^{\mathrm{A}}$ by introducing the converted production time $\left(T^{m^{*}}\right)$, which is shown in (2). Besides, arranging processes that require the same material on a 3D printer can get rid of material-change procedures, resulting in less time of set-up. The saved time can also be reflected in the converted setup time $\left(t c_{i}{ }^{m}\right)$. In this regard, $T^{m}$ is the actual time consumption while $T^{m^{*}}$ is introduced to obtain more efficient production 


$$
T^{m^{*}}=\sum_{i=1}^{k} T_{i}^{m}+\sum_{i=1}^{k} t c_{i}^{m}+\sum_{i=1}^{k-1} T\left(P_{i}^{m}, P_{i+1}^{m}\right)
$$

\subsubsection{Product cost}

The product cost is obtained by adding the material cost, transportation cost, energy-consumption cost, and machineoccupation cost together. The available time of A/SM machines is a kind of resource from the perspective of the platform operator. Therefore, the machine-occupation cost is related to the manufacturing time on the machine and the type of machines. The energy consumption of processing, set-up, transportation, and material consumption are all considered when calculating the energy-consumption cost. The product cost of task $m$ (denoted as $C^{m}$ ) is formulated in (3), where the first term indicates the material cost, the second term is the transportation cost, items 3-5 are the energy-consumption cost, and items 6-7 are the machine-occupation cost.

$$
C^{m}=\sum_{i=1}^{k} c m_{i}^{m}+\sum_{i=1}^{k-1} C\left(P_{i}^{m}, P_{i+1}^{m}\right)+\sum_{i=1}^{k} E_{i}^{m}+\sum_{i=1}^{k} e s_{i}^{m}+\sum_{i=1}^{k-1} e t_{i}^{m} E\left(P_{i}^{m}, P_{i+1}^{m}\right)+\sum_{i=1}^{k} c_{i}^{m} T_{i}^{m}+\sum_{i=1}^{k} c_{i}^{m} t s_{i}^{m}
$$

Similar to the discussions in the second paragraph of 5.1.1, the converted energy consumption during set-up is introduced (i.e. $e_{i}{ }^{m}$ ) to reflect the energy reduction brought by arranging the processing tasks with a similar temperature close to each other. The corresponding converted cost (denoted as $C^{m^{*}}$ ) is shown in (4).

$$
C^{m^{*}}=\sum_{i=1}^{k} c m_{i}^{m}+\sum_{i=1}^{k-1} C\left(P_{i}^{m}, P_{i+1}^{m}\right)+\sum_{i=1}^{k} E_{i}^{m}+\sum_{i=1}^{k} e c_{i}^{m}+\sum_{i=1}^{k-1} e t_{i}^{m} E\left(P_{i}^{m}, P_{i+1}^{m}\right)+\sum_{i=1}^{k} c_{i}^{m} T_{i}^{m}+\sum_{i=1}^{k} c_{i}^{m} t c_{i}^{m}
$$

5.2 The strategy for resource configuration on the platform

At the arrival of every new order/task, the resource configuration mechanism of the cloud platform can arrange the processes to proper A/SM resources. The general procedures can be divided into the following three stages.

(i) From the perspective of resource capability, a list of all resources that are capable of the required process is established for each process. These candidate resources meet the basic functional requirements of the orders.

(ii) From the perspective of resource availability, the unoccupied time slots of the candidate resources are obtained by inquiring their current task queue. These time slots can potentially undertake processing tasks on the platform.

(iii) From the perspective of resource efficiency and customers' satisfactory, processes of the received tasks are inserted into the time slots considering time, cost, quality, etc. The technique for order preference by similarity to ideal solution method (TOPSIS) is applied to select the final production plan for customers.

Based on the ontology model in Section 4, the resource selection in the first stage can be realized using techniques such as semantic matching (Zhang et al., 2017). A 7-tuple representation of processes is proposed in Section 5.2.1 to reflect the task queues of the A/SM resources and the resource configuration for each task. The tasks from customers are compiled into semi-structured files which are introduced in Section 5.2.2. The selection of production plans based on TOPSIS (Rao, 2013) is discussed in Section 5.2.3.

\subsubsection{Representation of the resource configuration}

For the convenience of resource configuration, a 7-tuple representation of processes is proposed in (5), where $l o c$ represents the location of the resources, $s s$ represents the starting time of set-up activities for this process, $s f$ represents the finish time of the set-up activities, $p s$ represents the starting time of processing, $p f$ represents the finish time of processing, $l s$ represents the starting time of the logistics activities, and $l f$ represents the finish time of the logistics activities.

$$
P_{i}^{m}(l o c, s s, s f, p s, p f, l s, l f)
$$


For example, a processing task after resource configuration can be expressed as (A01, 0, 2, 2, 26, 26, 34) - (A03, 35, $36,36,55,56,81)$ - (B21, 86, 88, 89, 107, 107, 140) - (User1, 141, 142, 142, 142, 142, 142), which means the product has been processed at three different locations (i.e., A01, A03, and B21) before delivered to the customer at time 142. The setup time of the three processes is 2, 1, 2 while the processing time is $24,19,18$. Besides, if the task queue of a machine is $(\mathrm{A} 03,3,4,4,37,37,50)$ - (A03, 35, 36, 37, 55, 56, 81) - (A03, 90, 94, 95, 130, 130, 160), then the available time slots of this machine range from 55 to 95 or any time after 130 .

\subsubsection{Semi-structured information of tasks and resources}

The semi-structured extensible mark-up language (XML) file is an ideal solution to process the information of tasks and machines for its simplicity of maintenance and wide application in computer programs. The information structure of tasks and resources is shown in Fig. 8.

The task information comes from orders from customers, where some values can be vague (i.e. provide a range instead of a precise value). During the processes of resource configuration, the unspecified values will be determined by the platform, thus narrowing down the solution space to obtain the exact configuration for each process. The example of the machine information model only shows part of the characteristics and current status of the manufacturing resource. Other characteristics discussed in Section 4 should also be included.

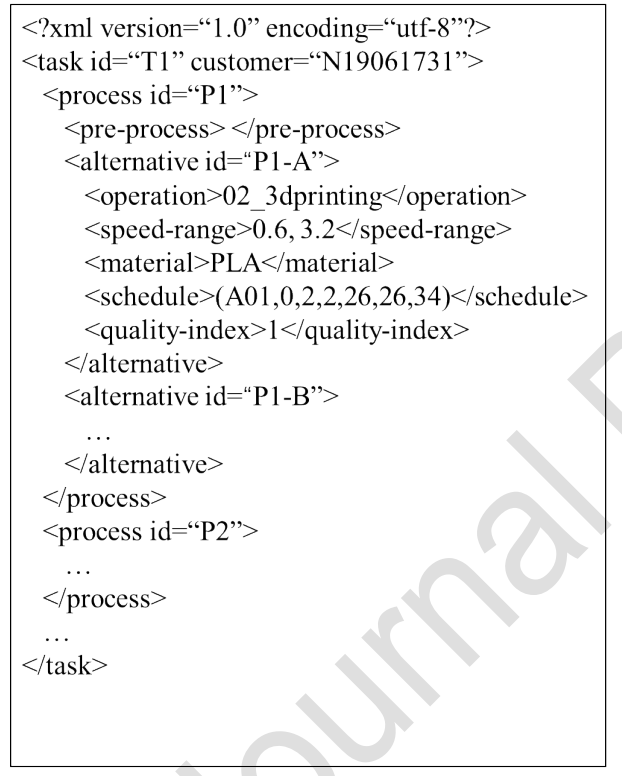

(a) Task information in the XML file

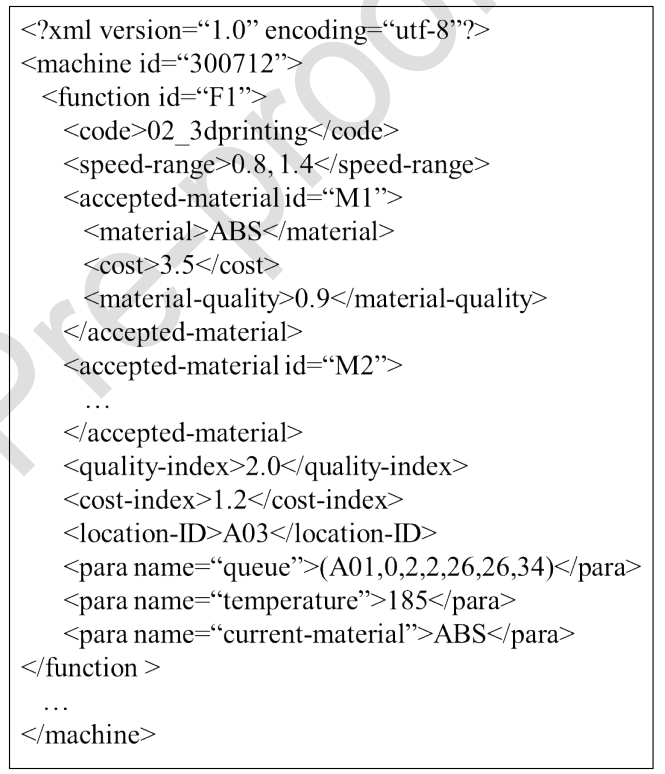

(b) Resource information in the XML file

Fig. 8 The information model of tasks and resources

\subsubsection{Plan selection based on TOPSIS}

After the first two stages of the resource configuration, it is possible to have more than one machine available for each process. The time, cost, and quality of all possible plans can be obtained according to the discussions in Section 5.1 . Then, combined with the weights on the three criteria (i.e. time, cost, and quality) given by customers, TOPSIS is used to select the optimal production plan. The procedures of TOPSIS is briefly introduced as follows.

(i) Normalize the decision table.

Table 2 shows the different time consumption, cost, and quality if applying different production plans. These values $\left(x_{i j}\right)$ need to be normalized according to (6), where $X_{i j}$ represents the normalized values.

$$
X_{i j}=\frac{x_{i j}-x_{j}^{\min }}{x_{j}^{\max }-x_{j}^{\min }}
$$

Table 2 Decision table of different processing routes with different time consumption, cost, and quality.

\begin{tabular}{llll}
\hline Processing route & Time & Cost & Quality \\
\hline
\end{tabular}


Plan B $\quad x_{21} \quad x_{22} \quad x_{23}$

Plan $n \quad x_{n 1} \quad x_{n 2} \quad x_{n 3}$

(ii) Form the weighted matrix.

A set of weights $w_{j}(j=1,2,3)$ such that $\Sigma j=1$ should be given by the customer, which reflects the relative importance of the three criteria. The weighted decision matrix (denoted as $\left[Y_{i j}\right]$ ) can be obtained by (7).

$$
\left[Y_{i j}\right]=\left[w_{j} X_{i j}\right]
$$

(iii) Calculate composite performance scores.

The ideal solution (denoted as $Y_{j}^{+}$) takes the shortest time, lowest cost, and highest quality from the elements in the weighted decision matrix, while the negative ideal solution (denoted as $Y_{j}^{-}$) takes the longest time, highest cost, and lowest quality, as formulated in (8) and (9).

$$
\begin{aligned}
& Y_{j}^{+}=\left\{X_{j=1}^{\min }, X_{j=2}^{\min }, X_{j=3}^{\max }\right\} \\
& Y_{j}^{-}=\left\{X_{j=1}^{\max }, X_{j=2}^{\max }, X_{j=3}^{\min }\right\}
\end{aligned}
$$

(iv) Calculate the separation to the ideal/negative ideal scores.

Euclidean distance is used to obtain the separation of each plan from the ideal one, which is formulated in (10) and (11), where $i=1,2, \ldots, n$.

$$
\begin{gathered}
d_{i}^{+}=\sqrt{\sum_{j=1}^{3}\left(X_{i j}-Y_{j}^{+}\right)} \\
d_{i}^{-}=\sqrt{\sum_{j=1}^{3}\left(X_{i j}-Y_{j}^{-}\right)}
\end{gathered}
$$

(v) Obtain the relative closeness to the ideal solution.

The relative closeness (denoted as $C_{i}$ ) of a specific plan to the ideal solution can be expressed by (12).

$$
C_{i}=\frac{d_{i}^{-}}{d_{i}^{-}+d_{i}^{+}}
$$

(vi) Sort the solutions (i.e. production plans) in descending order of the relative closeness.

The alternative plan with the highest closeness to the ideal plan is the optimal resource configuration under the given weights for different criteria.

\section{Illustrative case demonstration}

A prototype system was developed to test the feasibility of service encapsulation and cloud service operations, before being implemented in a real-life case. Two 3D printers and a turning/milling machine were used for the prototype system. First, the FDM 3D printers were configured with Repetier-Host (Repetier, 2016), which is a widely used software that allows operators to fully control most FDM 3D printers. Repetier supports B/S-based remote control and has provided some useful secondary development tools. So, it is feasible to construct the CMfg platform based on its related toolkits. Repetier-Server connects the 3D printers to a web server. Then, the vibration sensors and a web-camera were set up to capture the necessary information, which can be used by the maintenance team and displayed to customers. Data from the sensors and the 3D printer were transmitted to the server. Due to the limitations of the laboratory conditions, the turning/milling machine was not a $\mathrm{CNC}$ one, and its capability information was recorded manually and inputted to the server which may include delays. However, the electric current of the machine was monitored to determine whether it was 
working or not, which was basically sufficient for the demonstration. The tasks assigned to this turning/milling machine cannot be processed automatically and were displayed on a webpage designated as the Kanban for the machine operator, while in the real industry, some computer-aided engineering software is capable to generate codes for CNC machines according to the designs. A mobile application was also developed based on the Android SDK to demonstrate the workflow of CMfg, which is shown in Fig. 9.

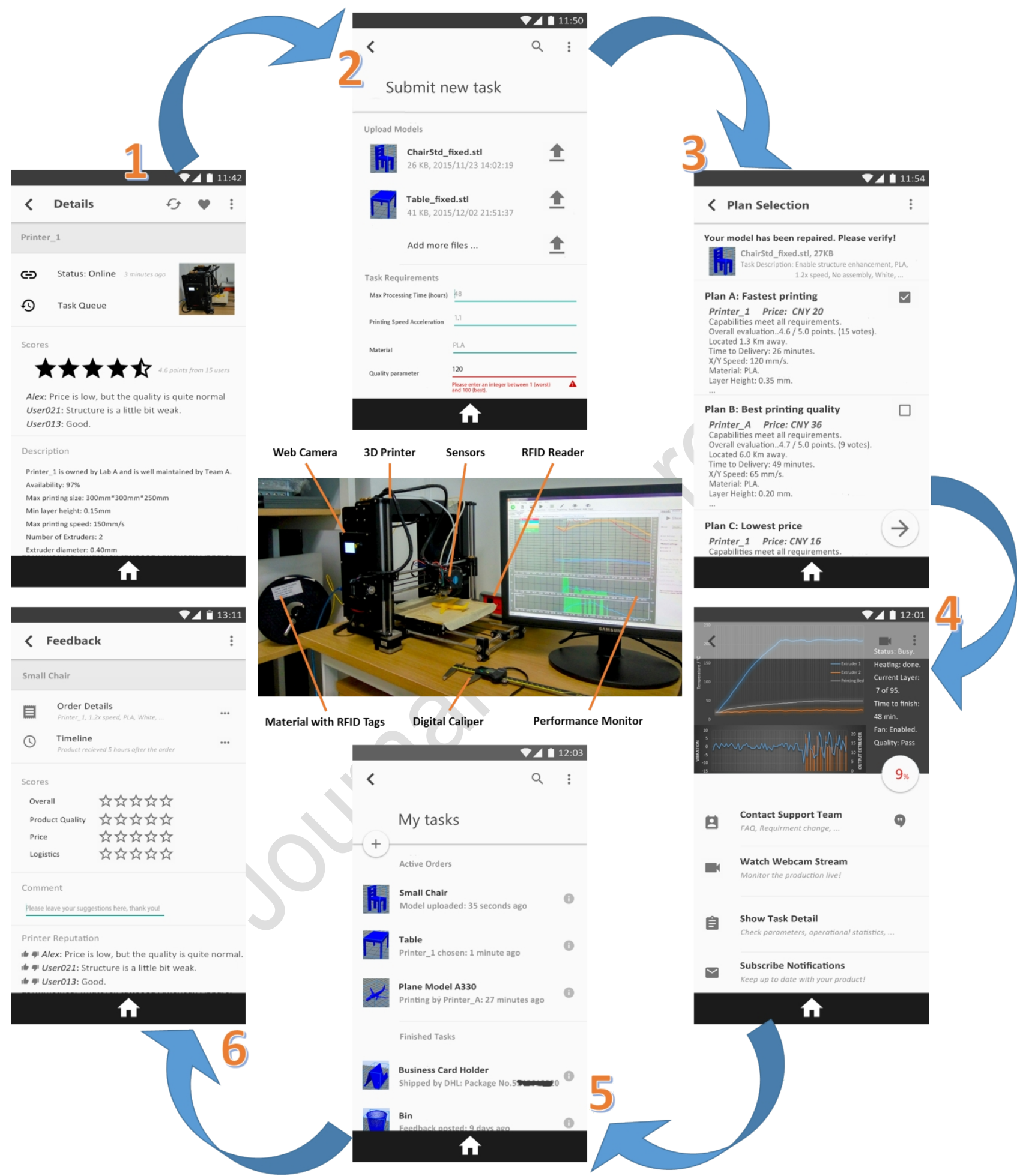

Fig. 9 The workflow of the cloud platform.

As described in Section 4, the 3D printer was encapsulated into many manufacturing services and they were registered at the server. Upon being connected to the cloud, related information of this printer could be accessed by customers (Step 1 in Fig. 9). Customers could upload the models via the mobile application. At the same time, they needed to specify the requirements for the task such as the material and quality standard (Step 2 in Fig. 9). The files were converted to the standard format and scanned for design errors. Meanwhile, the server kept collecting performance data from the three machines. By searching the task queue of each manufacturing resource, the two printers reported to the server that their 
status was unoccupied, and the capability properties met the requirement of the task. Based on the steps in Section 5.1.1, three production plans were generated, characterized by its shortest production time, best production quality, cheapest price, respectively. Customized plans were also possible to realize the trade-off among multiple objectives. After the customer's decision was made (i.e. to give relative weights to time, cost, and quality), and the payment was received, the optimal production plan was obtained. In this case, the task was added to the task queue of Printer_1 (Step 3 in Fig. 9). Customers can submit more tasks and the task assignment was carried out in the same way. During the manufacturing execution process, customers were able to watch the printing site live and had access to the data from different sensors (Step 4 in Fig. 9). The estimated task finishing time was also displayed in the application. The quality control service kept tracking the manufacturing processes and reported to the maintenance team if necessary. After printing the model, different parts of the model aircraft were assembled, and the supporting structures were removed by operators. The status of each task was reported to customers in real time (Step 5 in Fig. 9). Then, the product is packaged and delivered to the customer after inspection. The customer can trace the package through logistics information service, which will also be displayed in the application. Finally, the service was marked by the customer, and suggestions were also given that the layer height should be thinner for better quality (Step 6 in Fig. 9). This remark is stored in the database and will be analyzed by operating analysis service or technicians, so that production plans can be better designed. Historical remarks to different plans can also be viewed to customers.

The designed platform and applications were also demonstrated and tested in a last factory (i.e. manufacturers of the molds of shoes) in south China. Usually, they use injection molding equipment and CNC machines a lot for the shoe models. They started to apply 3D printers recently for fast prototyping during design to collect advice from customers by showing the printed models, which helped them to expand the market share a lot. They were interested in the designed platform as it can connect more kinds of printers and traditional subtractive manufacturing equipment such as a drilling machine. These shared A/SM resources may potentially help them to ease the financial pressure for buying all the required machines and to increase resource efficiency as much as possible. The details of the case are introduced below.
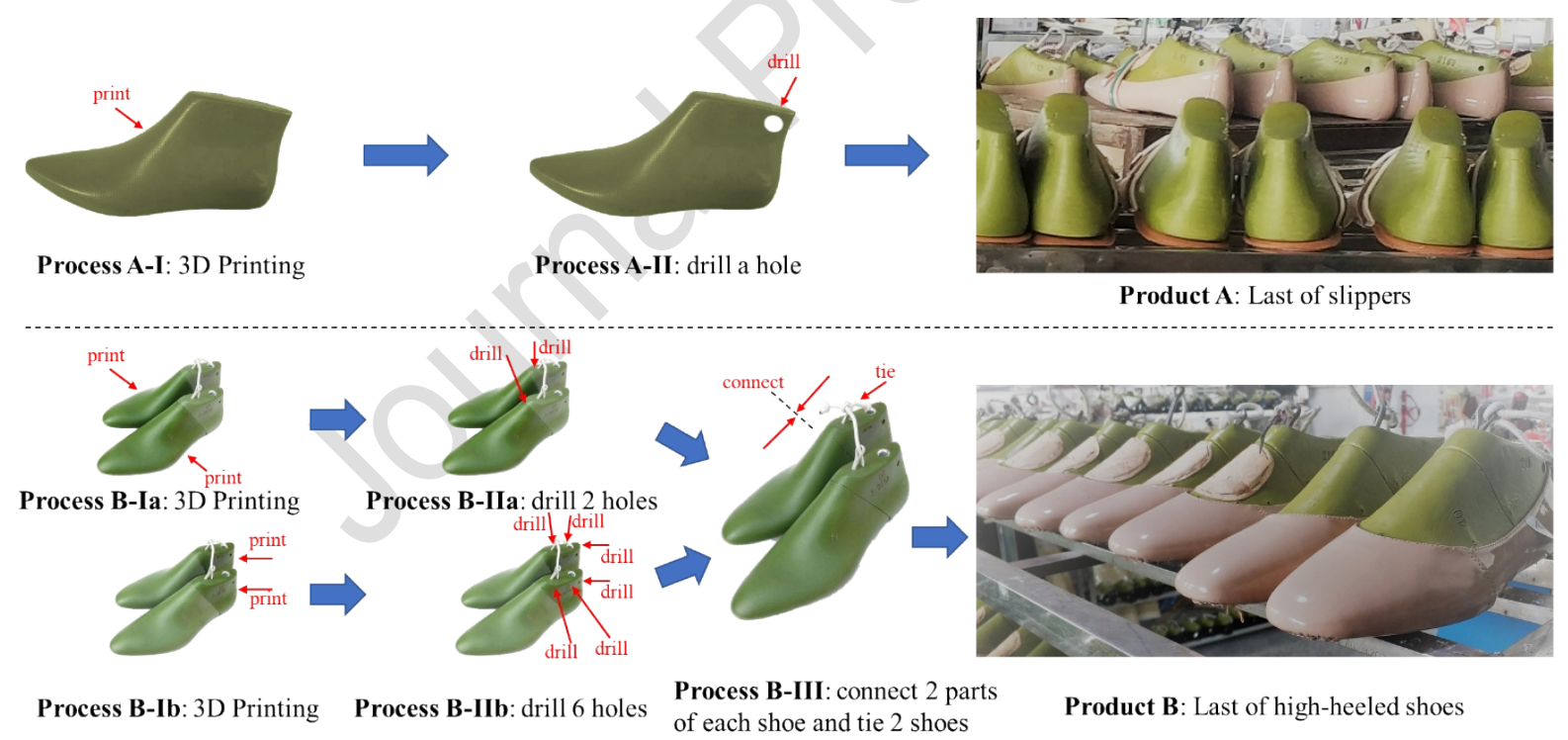

Fig. 10 Processing procedures for the two products in the last factory.

Fig. 10 presents two of their major products and the primary processing procedures using 3D printers. The final product of the slipper's last (i.e. Product A) is a single piece of mold, whose production involves the 3D printing of the overall shape and the drilling at the back-end. The final product of the last of high-heeled shoes (i.e. Product B) is a pair of separable lasts that can be adjusted for different shoe sizes. The two separable parts of each shoe are printed respectively before drilling and being connected to each other.

Currently, this factory has access to 3 3D printers, 2 drilling machines, and one working area of assembly for the two products, with the expected resource configuration shown in Fig. 11. The allowance time, set-up time, etc. have been included in the processing time of each process (because they did not have a record for the time consumption of every detailed action s). Some sensitive data (including the processing and delivery time) have been normalized in the Gantt 
chart. This factory needs to deliver 1 piece of Product $A$ and 2 pieces of Product B to Customer 1, while providing 5 pieces of Product A to Customer 2. The relative weights given by customers on time, cost, and quality are provided in Table 3 . The range of relative production speed, cost coefficient, and quality of machines are provided in Table 4. In Fig. 11, Blocks with slashes in the Gantt chart are the time slots that have been occupied by previously received tasks and cannot be adjusted. The 6 manufacturing resources and the 2 customers all located in a same industrial park for shoes. Thus, the logistics time between processes is combined with the set-up time that has already been included in the time consumption of the processes.

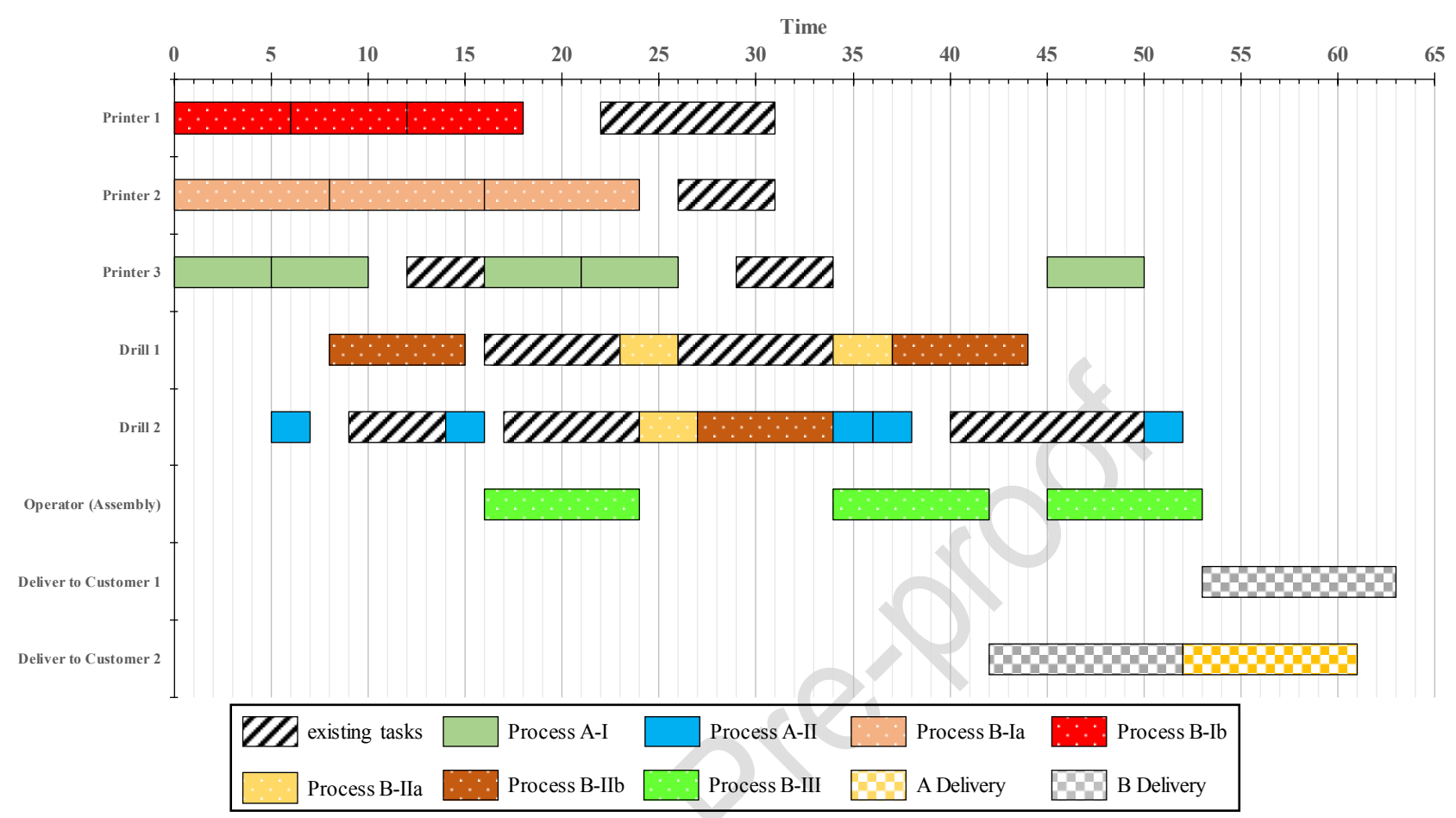

Fig. 11 Gantt chart of the currently expected resource configuration for this factory.

Table 3 Relative weights given by customers on different products.

\begin{tabular}{cccc}
\hline & Time & Cost & Quality \\
\hline Product A for Customer 1 & 0.2 & 0.4 & 0.4 \\
Product B for Customer 1 & 0.1 & 0.3 & 0.5 \\
Product A for Customer 2 & 0.3 & 0.6 & 0.1 \\
\hline
\end{tabular}

Table 4 Relative production speed, cost coefficient, and quality of machines.

\begin{tabular}{cccc}
\hline & Speed range & Cost coefficient & Quality \\
\hline 3D Printer 1 & $0.2-1.5$ & 1.75 & 0.92 \\
3D Printer 2 & $0.4-1.1$ & 0.99 & 0.85 \\
3D Printer 3 & $0.5-1.0$ & 0.78 & 0.71 \\
Drilling Machine 1 & 1.0 & 1.31 & 0.96 \\
Drilling Machine 2 & 0.9 & 1.02 & 0.86 \\
\hline
\end{tabular}

Table 5 Average power consumption (Watts) of the 3D printers in different working conditions.

\begin{tabular}{lccc}
\hline & Idling (Power dissipation) & Heating & Heating during printing \\
\hline 3D Printer 1 & 5 & 230 & 42 \\
3D Printer 2 & 3 & 175 & 27 \\
3D Printer 3 & 6 & 190 & 30 \\
\hline
\end{tabular}




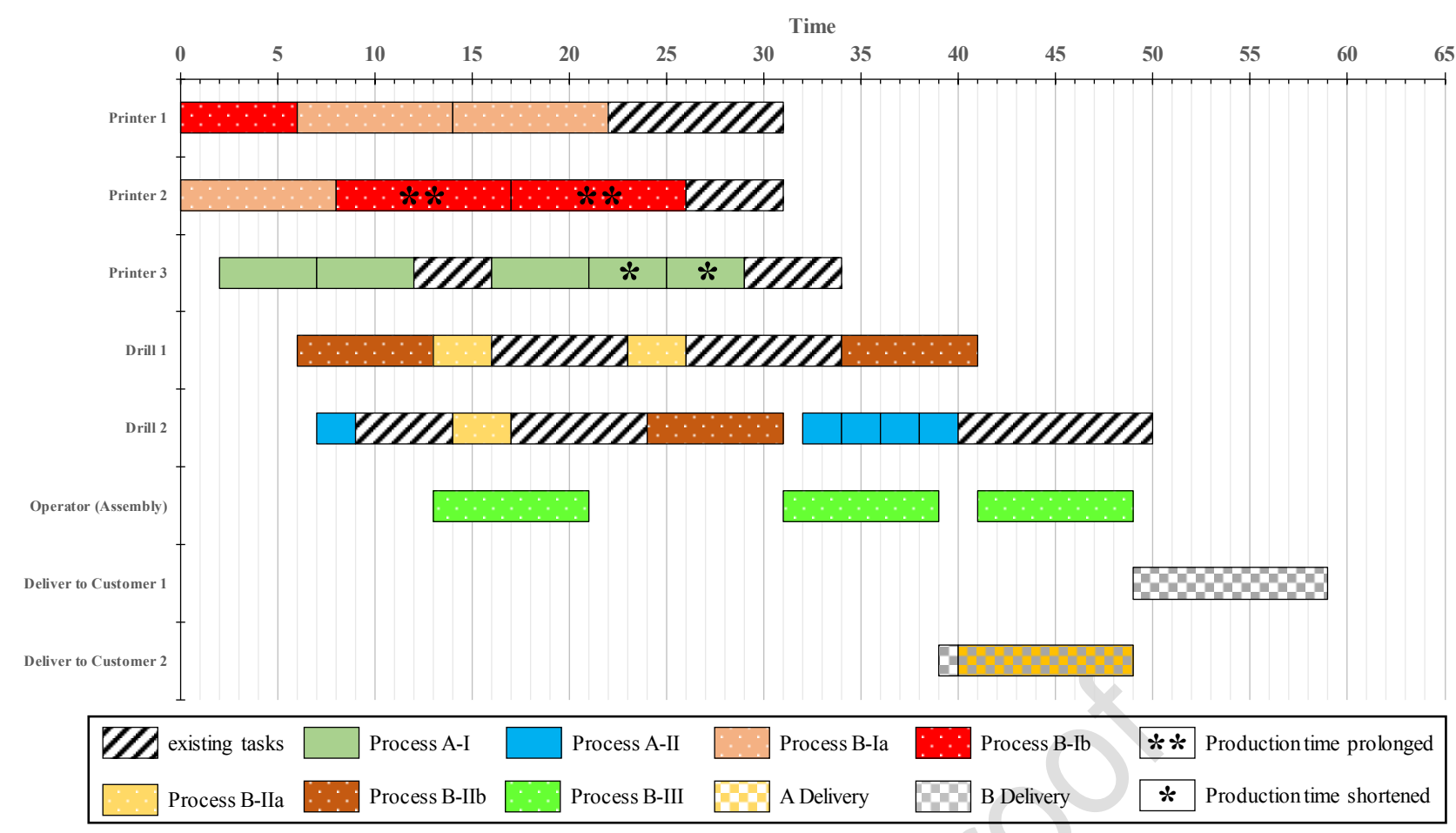

Fig. 12 Gantt chart of the resource configuration recommended by the cloud platform.

After some trial runs of the cloud platform, the resource configuration recommended by the platform was provided as shown in Fig. 12. Compared to the previous plan, some processing tasks were assigned to different machines. Besides, the processing time of 2 processes was prolonged while that of the other 2 processes was shortened. Since Customer 1 put greater weight on the quality of Product B, the production speed of Process B-Ib was decreased to improve the printing quality, so as to compensate for the degradation in quality standards when reassigning this process to Printer 2 rather than Printer 1. Besides, two processes (Process A-I) on Printer 3 were accelerated to fill the limited time slot. It is possible to accelerate their processing time by $20 \%$ because the required temperature for all tasks on this printer is consistent (i.e. $460 \mathrm{~K})$. Therefore, the set-up time (including waiting for the temperature to increase) can be greatly reduced.

By comparing Fig. 11 and Fig. 12, it is clear that the utilization rate of the 3 printers and 2 drilling machines were significantly improved. As discussed before, this could greatly reduce the times of heating, saving time and energy. Idling, heating, and printing are the primary working conditions of a typical FDM 3D printer. Idling power is consumed by the master control chips, the cooling fans, and multiple sensors on the 3D printer. Heating power is generally used to increase the temperature of the extruder and the heating bed. During printing, the majority of power goes to the step motors on three axes and the extruder, while others go to the heating system to maintain the temperature of the extruder and heating bed. According to Peng (2016), heating and melting is the primary power consumption during printing processes. To quantify the reduction in energy consumption of this research, the average power consumption related to heating of the 3 printers was measured in different working conditions (e.g., power dissipation during idling, power of heating, and heat preservation during printing) and presented in Table 5. The heating process from ambient temperature (i.e. $303 \mathrm{~K}$ ) to the printing temperature (i.e. $460 \mathrm{~K}$ for the extruder and $333 \mathrm{~K}$ for the heating bed) roughly took 0.5 -unit time, while the temperature of the extruder and heating bed roughly decreases $80 \mathrm{~K}$ and $10 \mathrm{~K}$ per unit time when the printer is idling. All the printing parts used the same material (i.e. polylactic acid) and required the same printing temperature. The estimated energy consumption (from the beginning of the first task to the end of the last task) related to heating for the 3 printers were calculated in Table 6. Although energy consumption was not directly considered by customers when placing the order, the heating energy consumption of the 3 printers still decreased from 3686.1 (J*unit-time/s) to 3347.0 ( $\mathrm{J} *$ unit-time/s), i.e. $9.2 \%$ reduction.

Table 6 Estimated energy consumption for heating (J*unit-time/s) of the 3D printers. 


\begin{tabular}{|c|c|c|c|}
\hline & Resource & Energy & Ournal pre-proot \\
\hline & configuration & consumption & Calculations \\
\hline 3D Printer 1 & as in Fig. 11 & 1328.5 & $Q_{1}=230 \mathrm{~W} \times 0.5+42 \mathrm{~W} \times 17.5+5 \mathrm{~W} \times 4+230 \mathrm{~W} \times 0.5+42 \mathrm{~W} \times 8.5$ \\
\hline 3D Printer 1 & as in Fig. 12 & 1396.0 & $Q_{1}^{\prime}=230 \mathrm{~W} \times 0.5+42 \mathrm{~W} \times 30.5$ \\
\hline 3D Printer 2 & as in Fig. 11 & 929.6 & $Q_{2}=175 \mathrm{~W} \times 0.5+27 \mathrm{~W} \times 23.5+3 \mathrm{~W} \times 2+175 \mathrm{~W} \times 0.45+27 \mathrm{~W} \times 4.55$ \\
\hline 3D Printer 2 & as in Fig. 12 & 911.0 & $Q_{2}^{\prime}=175 \mathrm{~W} \times 0.5+27 \mathrm{~W} \times 30.5$ \\
\hline 3D Printer 3 & as in Fig. 11 & 1428.0 & $\begin{aligned} Q_{3}= & 190 \mathrm{~W} \times 0.5+30 \mathrm{~W} \times 9.5+6 \mathrm{~W} \times 2+190 \mathrm{~W} \times 0.45+30 \mathrm{~W} \times 13.55+6 \mathrm{~W} \\
& \times 3+190 \mathrm{~W} \times 0.5+30 \mathrm{~W} \times 4.5+6 \mathrm{~W} \times 11+190 \mathrm{~W} \times 0.5+30 \mathrm{~W} \times 4.5\end{aligned}$ \\
\hline 3D Printer 3 & as in Fig. 12 & 1040.0 & $Q_{3}{ }^{\prime}=190 \mathrm{~W} \times 0.5+30 \mathrm{~W} \times 31.5$ \\
\hline
\end{tabular}

It can be concluded from the case demonstration that CMfg has brought a lot of advantages to customers, platform operators, and service providers. In this mode, manufacturing information and quality standards are more transparent to customers. Customers will have more choices and can make better decisions according to this information. For platform operators, they will have better tools (services) to help with their work. Also, they may get valuable statistics of users' preference for better business and potentially greater profits (provided they are granted by customers). As for service providers, the platform can provide feedback from customers, and can further increase their competitiveness. According to the case study, it is possible to increase the utilization rate of manufacturing resources while reducing unnecessary energy consumption in practice, which is good for both economic and social benefits. From the environmental perspective, the cloud-based A/SM platform integrates many kinds of manufacturing resources and may reduce lots of unnecessary purchases of them. The optimization strategies used in the operating procedures have factored in the distance between distributed resources which is reflected in the total cost and time, so the customers no longer need to travel a lot to find suitable resources, reducing much emission. The pricing models can also be used to encourage production plans with less environmental impact.

\section{Conclusions and outlook}

CMfg is a service-oriented manufacturing model and integrates manufacturing resources in a plug-and-play fashion. Although CMfg has been studied for years, its commercial implementations are still quite limited. Recently, the rapid development of AM has brought about an unprecedented opportunity to implement CMfg. As 3D printers are widely used for rapid prototyping, the manufacturing batch is usually small. This single-step fabrication method has decreased the complexity of implementing CMfg. Also, many owners of 3D printers are individuals. As a result, the utilization rate of $3 \mathrm{D}$ printers is usually low. CMfg is a perfect solution to increase the resource efficiency of AM. The idea of integrating additive and subtractive manufacturing on CMfg platform creates the opportunity to compromise the merits of both methods and increase the capabilities of CMfg.

In this research, a cloud platform is designed to integrate both additive and subtractive manufacturing resources from the whole society, increasing the utilization rate of the individual resources while reducing the energy consumption. The modeling of cloud-based manufacturing services was introduced. Both additive and subtractive manufacturing resources were encapsulated into shared web services in a similar procedure, so that they can be accessed and invoked easily. The typical parameters of 3D printers were listed for the modeling of AM services. The collaborative CMfg platform was designed to publish, search, invoke A/SM services, and to match them with tasks. The A/SM services, auxiliary manufacturing services, etc. can all be published to the platform. The business and manufacturing processes for cloudbased A/SM were also discussed. In addition, some improved strategies for resource configuration on the platform were modeled and proposed considering production time, cost, and QoS. The environmental impact was also considered throughout service modeling. Some case scenarios were analyzed to demonstrate the proposed models and test their feasibility. The improved utilization rate of A/SM resources and the reduction in energy consumption were discussed and calculated. Besides, the advantages of the proposed CMfg platform are discussed according to the case analysis. The cloudbased mode can reduce the unnecessary consumption of A/SM resources and the energy for heating and melting the material; the customers will have more options on their desired products within the budget and the production can be more flexible, etc. 
In order to promote the implementation of CMfg plattforms with higher resource efficiency, the future works need to focus on the modeling of lifecycle energy consumption of different resources and activities on the cloud platform. Also, the resource matching mechanism should be further improved when the type and quantity of resource increases in the future.

\section{Acknowledgment}

This research was supported in part by the National Natural Science Foundation of China (51675441), in part by the Seed Foundation of Innovation and Creation for Graduate Students in Northwestern Polytechnical University (Z2017014), and in part by the 111 Project Grant of NPU (B13044). The authors would like to thank the editors and reviewers for their dedication to this research. We also acknowledge the opportunities of on-site surveys brought by the Department of Mechanical and Energy Engineering, Southern University of Science and Technology (China). We appreciate the valuable suggestions on this research from Dr. Ying Liu and Prof. Ou Tang.

\section{References}

Argoneto, P., Renna, P., 2016. Supporting capacity sharing in the cloud manufacturing environment based on game theory and fuzzy logic. Enterprise Information Systems, 10(2), 193-210. https://doi.org/10.1080/17517575.2014.928950.

Ameri, F., McArthur, C., 2014. Semantic rule modelling for intelligent supplier discovery. International Journal of Computer Integrated Manufacturing. 27(6), 570-590. https://doi.org/10.1080/0951192X.2013.834467.

Bhattacharya, A., Tiwari, M. K., Harding, J. A., 2012. A framework for ontology based decision support system for elearning modules, business modeling and manufacturing systems. Journal of Intelligent Manufacturing. 23(5), $1763-$ 1781. https://doi.org/10.1007/s10845-010-0480-6.

Brant, A., Sundaram, M. M., 2015. A novel system for cloud-based micro additive manufacturing of metal structures. Journal of Manufacturing Processes. 20, 478-484. https://doi.org/10.1016/j.jmapro.2015.06.020.

Buckholtz, B., Ragai, I., Wang., L., 2015. Cloud Manufacturing: Current Trends and Future Implementations. Journal of Manufacturing Science and Engineering. 137(4), 040902. https://doi.org/10.1115/1.4030009.

Caggiano, A., 2018. Cloud-based manufacturing process monitoring for smart diagnosis services. International Journal of Computer Integrated Manufacturing. 31(7), 612-623. https://doi.org/10.1080/0951192X.2018.1425552.

Chard, K., Bubendorfer, K., Caton, S., Rana, O. F., 2012. Social cloud computing: A vision for socially motivated resource sharing. IEEE Transactions on Services Computing. 5(4), $551-563$.

Chen, W., Paik, I., 2013. Improving efficiency of service discovery using Linked data-based service publication. Information Systems Frontiers. 15(4), 613-625. https://doi.org/10.1007/s10796-012-9381-x.

Chua, C. K., Leong, K. F., 2014. 3D Printing and Additive Manufacturing: Principles and Applications. World Scientific Publishing Company Pte Limited. https://doi.org/10.1142/10200.

Curbera, F., Duftler, M., Khalaf, R., Nagy, W., Mukhi, N., Weerawarana, S., 2002. Unraveling the Web services web: an introduction to SOAP, WSDL, and UDDI. IEEE Internet Computing. 6(2), 86-93. https://doi.org/10.1109/4236.991449.

Dadzie, A. S., Bhagdev, R., Chakravarthy, A., Chapman, S., Iria, J., Lanfranchi, V., et al., 2009. Applying semantic web technologies to knowledge sharing in aerospace engineering. Journal of Intelligent Manufacturing. 20(5), 611-623.

Du, W., Bai, Q., Zhang, B., 2016. A novel method for additive/subtractive hybrid manufacturing of metallic parts. Procedia Manufacturing. 5, 1018-1030. https://doi.org/10.1016/j.promfg.2016.08.067.

Govindan, K., Diabat, A., Shankar, K. M., 2015. Analyzing the drivers of green manufacturing with fuzzy approach. Journal of Cleaner Production. 96(1), 182-193. https://doi.org/10.1016/j.jclepro.2014.02.054.

IBM developerWorks, 2002. Understanding UDDI. https://www.ibm.com/developerworks/cn/webservices/ws-featuddi/ (accessed 30 August 2018, in Chinese).

Jang, J., Jeong, B., Kulvatunyou, B., Chang, J., Cho, H., 2008. Discovering and integrating distributed manufacturing services with semantic manufacturing capability profiles. International Journal of Computer Integrated Manufacturing. 21(6), 631-646. https://doi.org/10.1080/09511920701350920. 
Kang, S., Patil, L., Rangarajan, A., Moitra, A., Jia,T., Rôbinson, D., etâl., 2015. Extraction of manufacturing rules from unstructured text using a semantic framework. ASME 2015 International Design Engineering Technical Conferences and Computers and Information in Engineering Conference. 1B, V01BT02A033. https://doi.org/10.1115/DETC2015$\underline{47556}$.

Le, V. T., Paris, H., Mandil, G., 2017. Process planning for combined additive and subtractive manufacturing technologies in a remanufacturing context. Journal of Manufacturing Systems. 44, 243-254. https://doi.org/10.1016/j.jmsy.2017.06.003.

Lee, J., Kao, H. A., Yang, S., 2014. Service innovation and smart analytics for Industry 4.0 and big data environment. Procedia CIRP. 16, 3-8. https://doi.org/10.1016/j.procir.2014.02.001.

Lee, J. Y., Tan, W. S., An, J., Chua, C. K., Tang, C. Y., Fane, A. G., et al., 2016. The potential to enhance membrane module design with 3D printing technology. Journal of Membrane Science. 499, 480-490.

Li, C. Z., Zhong, R. Y., Xue, F., Xu, G., Chen, K., Huang, G. G., et al., 2017. Integrating RFID and BIM technologies for mitigating risks and improving schedule performance of prefabricated house construction. Journal of Cleaner Production. 165, 1048-1062. https://doi.org/10.1016/j.jclepro.2017.07.156.

Liu, Y., Wang, L., Wang, X. V., 2018. Cloud manufacturing: latest advancements and future trends. Procedia Manufacturing. 25, 62-73. https://doi.org/10.1016/j.promfg.2018.06.058.

Ma, S., Zhang, Y., Lv, J., Yang, H., Wu, J., 2019. Energy-cyber-physical system enabled management for energy-intensive manufacturing industries. Journal of Cleaner Production, 226, 892-903. https://doi.org/10.1016/j.jclepro.2019.04.134

Mai, J., Zhang, L., Tao, F., Ren, L., 2016. Customized production based on distributed 3D printing services in cloud manufacturing. International Journal of Advanced Manufacturing Technology. 84(1-4), 71-83. https://doi.org/10.1007/s00170-015-7871-y.

Meier, H., Roy, R., Seliger, G., 2010. Industrial product-service systems-IPS2. CIRP Annals-Manufacturing Technology. 59(2), 607-627. https://doi.org/10.1016/j.cirp.2010.05.004.

Newman, S. T., Zhu, Z., Dhokia, V., Shokrani, A., 2015. Process planning for additive and subtractive manufacturing technologies. CIRP Annals. 64(1), 467-470. https://doi.org/10.1016/j.cirp.2015.04.109.

Paris, H., Mokhtarian, H., Coatanea, E., Museau, M., Ituarte, I. F., 2016. CIRP Annals. 65(1), $29-32$. https://doi.org/10.1016/j.cirp.2016.04.036.

Peng, T., 2016. Analysis of energy utilization in 3D printing processes. Procedia CIRP. 40, 62-67. https://doi.org/10.1016/j.procir.2016.01.055.

Priarone, P. C., Ingarao, G., 2017. Towards criteria for sustainable process selection: On the modelling of pure subtractive versus additive/subtractive integrated manufacturing approaches. Journal of Cleaner Production. 144, 57-68. https://doi.org/10.1016/j.jclepro.2016.12.165.

Rahim, S. L., Maidin, S., 2014. Feasibility study of additive manufacturing technology implementation in Malaysian automotive industry using analytic hierarchy process. Advanced Materials Research. 903, 450-454.

Rao, R. V., 2013. Decision making in the manufacturing environment using graph theory and fuzzy multiple attribute decision making methods. Springer Science \& Business Media. https://doi.org/10.1007/978-1-4471-4375-8.

Ren, L., Zhang, L., Zhao, C., Chai, X., 2013. Cloud manufacturing platform: Operating paradigm, functional requirements, and architecture design. ASME 2013 International Manufacturing Science and Engineering Conference collocated with the 41st North American Manufacturing Research Conference. 2, V002T02A009. https://doi.org/10.1115/MSEC2013-1185.

Repetier, 2016. Repetier-Host Documentation. https://www.repetier.com/ (accessed 30 August 2018)

Rudolph, J. P., Emelmann, C. 2017. A Cloud-based platform for automated order processing in additive manufacturing. Procedia CIRP. 63, 412-417. https://doi.org/10.1016/j.procir.2017.03.087.

Simeone, A., Caggiano, A., Boun, L., Deng, B., 2019. Intelligent cloud manufacturing platform for efficient resource sharing in smart manufacturing networks. Procedia CIRP. 79, 233-238. https://doi.org/10.1016/j.procir.2019.02.056.

Simeone, A., Caggiano, A., Deng, B., Zeng, Y., Boun, L., 2018. Resource efficiency optimization engine in smart production networks via intelligent cloud manufacturing platforms. Procedia CIRP. 78, 19-24. https://doi.org/10.1016/j.procir.2018.10.003.

Stanford Center for Biomedical Informatics Research, 2016. Protégé. https://protege.stanford.edu/ (accessed 30 August 
Stansbury, J. W., Idacavage, M. J., 2016. 3D printing with polymers: Challenges among expanding options and opportunities. Dental Materials. 32(1), 54-64.

Tao, F., Cheng, Y., Xu, L., Zhang, L., Li, H., 2014. CCIoT-CMfg: cloud computing and Internet of Things-based cloud manufacturing service system. IEEE Transactions on Industrial Informatics. 10(2), 1435-1442. https://doi.org/10.1109/TII.2014.2306383.

Tao, F., Zhang, L., Liu, Y., Cheng, Y., Wang, L., Xu, X., 2015. Manufacturing service management in cloud manufacturing: overview and future research directions. Journal of Manufacturing Science and Engineering.137(4), 040912. https://doi.org/10.1115/1.4030510.

Valilai, O., Houshmand, M., 2013. A collaborative and integrated platform to support distributed manufacturing system using a service-oriented approach based on cloud computing paradigm. Robotics and Computer Integrated Manufacturing. 29(1), 110-127.

Wang, L., Wang., X. V., Gao, L., Vancza, J., 2014. A cloud-based approach for WEEE remanufacturing. CIRP Annals. 63(1), 409-412. https://doi.org/10.1016/j.cirp.2014.03.114.

Watson, J. K., Taminger, K. M. B., 2018. A decision-support model for selecting additive manufacturing versus subtractive manufacturing based on energy consumption. Journal of Cleaner Production. 176, 1316-1322. https://doi.org/10.1016/j.jclepro.2015.12.009.

Wu, D., Greer, M. J., Rosen, D. W., Schaefer, D., 2013. Cloud manufacturing: Strategic vision and state-of-the-art. Journal of Manufacturing Systems. 32(4), 564-579. https://doi.org/10.1016/j.jmsy.2013.04.008.

Xu, X., 2012. From cloud computing to cloud manufacturing. Robotics and Computer-Integrated Manufacturing. 28(1), 75-86. https://doi.org/10.1016/j.rcim.2011.07.002.

Zhang, L., Luo, Y., Tao, F., Ren, L., Zhang, X., Guo, H., et al., 2014. Cloud manufacturing: A new manufacturing paradigm, Enterprise Information Systems. 2(2), 167-187. https://doi.org/10.1080/17517575.2012.683812.

Zhang, Y., Xi, D., Li, R., Sun, S., 2016. Task-driven manufacturing cloud service proactive discovery and optimal configuration method. International Journal of Advanced Manufacturing Technology. 84(1-4), 29-45. https://doi.org/10.1007/s00170-015-7731-9.

Zhang, Y., Zhang, G., Liu, Y., Hu, D., 2017. Research on services encapsulation and virtualization access model of machine for cloud manufacturing, Journal of Intelligent Manufacturing. 28(5), 1109-1123. https://doi.org/10.1007/s10845-015-1064-2.

Zhang, Y., Zhang, G., Wang, J., Sun, S., Si, S., Yang, T., 2014. Real-time information capturing and integration framework of the internet of manufacturing things. International Journal of Computer Integrated Manufacturing. 28(8), 811-822. https://doi.org/10.1080/0951192X.2014.900874.

Zheng, P., Lin, T. J., Chen, C. H., Xu, X., 2018. A systematic design approach for service innovation of smart productservice systems. Journal of Cleaner Production, 201, 657-667. https://doi.org/10.1016/j.jclepro.2018.08.101.

Zhu, Z., Anwer, N., Huang, Q., Mathieu, L., 2018. Machine learning in tolerancing for additive manufacturing. CIRP Annals. 67(1), 157-160. https://doi.org/10.1016/j.cirp.2018.04.119. 


\section{A cloud service platform integrating additive and subtractive manufacturing with}

\section{high resource efficiency}

Highlights:

1. A cloud-based manufacturing platform was proposed where additive and subtractive manufacturing resources can be wrapped as equivalent services that can be invoked by customers

2. The modeling of additive manufacturing resources was illustrated by selecting the key parameters of a Fused Deposition Modeling 3D printer

3. Some improved strategies for the resource configuration on the platform were proposed that considered the time consumption for both production and logistics, the energy waste during the change of tasks, etc.

4. A prototype application was developed and tested in a factory, where the increased utilization rate of machines and the reduced energy waste during heating were observed. 